\title{
The development of the N1 and N2 components in auditory oddball paradigms: a systematic review with narrative analysis and suggested normative values
}

\author{
David Tomé • Fernando Barbosa $\cdot$ Kamila Nowak • \\ João Marques-Teixeira
}

\begin{abstract}
Auditory event-related potentials (AERPs) are widely used in diverse fields of today's neuroscience, concerning auditory processing, speech perception, language acquisition, neurodevelopment, attention and cognition in normal aging, gender, developmental, neurologic and psychiatric disorders. However, its transposition to clinical practice has remained minimal. Mainly due to scarce literature on normative data across age, wide spectrum of results, variety of auditory stimuli used and to different neuropsychological meanings of AERPs components between authors. One of the most prominent AERP components studied in last decades was N1, which reflects auditory detection and discrimination. Subsequently, N2 indicates attention allocation and phonological analysis. The simultaneous analysis of $\mathrm{N} 1$ and $\mathrm{N} 2$ elicited by feasible novelty experimental paradigms, such as auditory oddball, seems an objective method to assess central auditory processing. The aim of this systematic review was to bring forward normative values for auditory oddball $\mathrm{N} 1$ and N2 components across age. EBSCO, PubMed, Web of Knowledge and Google Scholar were systematically searched for studies that elicited N1 and/or N2 by auditory oddball paradigm. A total of 2,764 papers were initially identified in
\end{abstract}

D. Tomé $(\bowtie)$

Department of Audiology, School of Allied Health Sciences, Polytechnic Institute of Porto, Porto, Portugal

e-mail: dts@estsp.ipp.pt

D. Tomé · F. Barbosa · J. Marques-Teixeira Laboratory of Neuropsychophysiology, Faculty of Psychology and Educational Sciences, University of Porto,

Porto, Portugal

K. Nowak

Laboratory of Neuropsychology, Nencki Institute of Experimental Biology, Polish Academy of Sciences, Warsaw, Poland the database, of which 19 resulted from hand search and additional references, between 1988 and 2013, last 25 years. A final total of 68 studies met the eligibility criteria with a total of 2,406 participants from control groups for $\mathrm{N} 1$ (age range 6.6-85 years; mean 34.42) and 1,507 for $\mathrm{N} 2$ (age range 9-85 years; mean 36.13). Polynomial regression analysis revealed that $\mathrm{N} 1$ latency decreases with aging at $\mathrm{Fz}$ and $\mathrm{Cz}, \mathrm{N} 1$ amplitude at $\mathrm{Cz}$ decreases from childhood to adolescence and stabilizes after 30-40 years and at Fz the decrement finishes by 60 years and highly increases after this age. Regarding N2, latency did not covary with age but amplitude showed a significant decrement for both $\mathrm{Cz}$ and Fz. Results suggested reliable normative values for $\mathrm{Cz}$ and $\mathrm{Fz}$ electrode locations; however, changes in brain development and components topography over age should be considered in clinical practice.

Keywords Event-related potentials - Auditory oddball paradigm $\cdot \mathrm{N} 1$ wave $\cdot \mathrm{N} 2$ wave $\cdot$ Aging $\cdot$ Normative values

\section{Introduction}

As a researcher of the third generation of neuroscientists, we are in the course of explaining the possession of psychological attributes by human beings, probably obsessively ascribing such attributes not to the mind but to the brain or parts of the brain. This is a rising issue among neurophilosophers, we still cannot explain how we perceive or think by reference to the brain or some part of the brain, for it makes no sense to ascribe such psychological attributes to anything less than a human being as a whole (Bennett and Hacker 2007).

Thereupon, systematic reviews are required to update, appraise and synthesize all quality research evidence relevant to a question. Such method is necessary and essential 
in today's neuroscience, cognitive psychology and psychophysiological research.

Keeping the above in mind, ever since Berger (1929) demonstrated that it is possible to record the electrical activity of the brain by placing electrodes on the surface of the scalp and with $3 / 4$ of century of knowledge since the first investigation of sound-evoked changes in the electroencephalogram (EEG) in the waking human brain (Davis 1939), there has been always considerable interest in the relationship between these recordings of neurophysiological activity and psychological processes in various fields and types of studies (e.g., experimental, translational, clinical, case study). We will review one of the most prominent human auditory event-related potential and field (ERP and ERF, respectively) - the N1/N1 m.

The electrical activity occurring on the scalp consists of changing electrical voltages that are caused by action potentials summed over large numbers of neurons, synapses, neuronal pathways and systems. There are two non-invasive measures for this electrical brain activity, the EEG and ERPs, both in time course. The EEG measures the spontaneous electrical brain activity and the ERPs are derived by averaging EEG changes over experimental or cognitive events. The averaging process attenuates the spontaneous activity in the EEG and results in electrical potential changes related to specific events (Eysenck and Keane 2003).

ERPs are induced exogenously by environmental events (such as sensory stimuli) or endogenously by processes such as decision making, eliciting a characteristic series of waves labeled according to their latency and polarity (Davis and Zerlin 1966). Parallel streams of neuronal activity originate overlapping ERP deflections composed of several components. Therefore, a component can be defined as a voltage contribution to the ERP which reflects a functionally discrete stage of neuronal processing occurring in a restricted cerebral area (Näätänen and Picton 1987), also are hypothesized to be linked and reflected by psychological and cognitive processes (Hillyard and Kutas 1983; Fabiani et al. 2007).

In auditory novelty processing experiments, the experimental paradigm that seems the most feasible is the oddball paradigm. The oddball paradigm, as a signal-detection paradigm was first described by Ritter and Vaughan (1969). In the auditory modality, it was first used in 1975 by Squires, Squires and Hillyard at the University of California, San Diego, USA (Squires et al. 1975). In this paradigm, physically deviant stimuli (silence included) are randomly presented among repetitive streams of homogenous sounds (standard stimuli; Näätänen 1975; Näätänen et al. 1978; Donchin 1981; Jääskeläinen 2012).

The electrically recorded N1 or N100 in auditory oddball stimulation is a negative wave response, which peaks about $100 \mathrm{~ms}$ after stimulus onset and lasts for approximately 100 ms (Näätänen and Picton 1987; Tiitinen et al. 1994; May and Tiitinen 2010). Multiple sources have been identified to generate N1 wave.

In the classical review of Näätänen and Picton (1987), it was concluded that at least six different cerebral processes, occurring in different cerebral locations and subserving different psychophysiological functions, can contribute to a negative wave recorded from the scalp peaking between 50 and $150 \mathrm{~ms}$ : (1) a component generated bilaterally in the auditory cortex by vertically oriented sources in the supratemporal plane (STP); (2) a component generated in the association cortex on the lateral aspect of the superior temporal gyrus (STG) and parietal cortex, also termed by T-complex (Wolpaw and Penry 1975); (3) a vertex negative component generated in the motor and premotor cortices, its widespread and nonspecific neuronal networks with thalamo-reticular system facilitates stimulus detection, analysis and response (Crowley and Colrain 2004); (4) the mismatch negativity (MMN); (5) a temporal component of the processing negativity; and a (6) frontal component of the processing negativity. The first three are considered the true N1 components. Thus, $\mathrm{N} 1$ is a fronto-centrally component with a peak latency of $100 \mathrm{~ms}$ after stimulus onset generated bilaterally mainly in the auditory cortices. Its magnetic equivalent (N1 m/N100 m; Elberling et al. 1980; Hari et al. 1980) originates deep within the Sylvian fissure in tonotopically organized areas (Yamamoto et al. 1988; Cansino et al. 1994), but also comprises secondary areas such as Heschl's gyrus, STG and planum temporale providing the major source (Papanicolaou et al. 1990; Pantev et al. 1995; Jääskeläinen et al. 2004; Inui et al. 2006).

The auditory ERP N1, does not reflect a single underlying cerebral process, but it appears to contain both stimulus-specific and stimulus-nonspecific components, closely following the P1 component (Yvert et al. 2001; Hamm et al. 2013). These ERP components reflect sensory and perceptual processes. Further, N1 seems to reflect early synchronization between primary and secondary auditory cortices in the lateral and STP (Yvert et al. 2005; Liasis et al. 2006).

The generation source location of N1 depends on stimulus frequency. Woods et al. (1993) have found that $\mathrm{N} 1$ is more frontally distributed following $4,000 \mathrm{~Hz}$ than $250 \mathrm{~Hz}$ tone burst stimuli. Numerous studies have shown that N1 amplitude and latency have a large variation according to type of deviant auditory stimuli. For example, in speech oddball paradigms, the first stages of detection, graphemic analysis and stimuli recognition correspond to the appearance of a N1 with latency between 150 and $200 \mathrm{~ms}$ (Bentin and Carmon 1984). Subsequently, the N2 indicates an attentional allocation and stage of phonological type analysis of the information, related to the sounds of the language, both in the auditory and visual modalities of 
presentation (Sams et al. 1985; Reinvang et al. 2000). Two components contribute to forming N2, usually labeled MMN (N2a) and N2b (Novak et al. 1990, 1992). The first component (MMN) has been related to the automatic detection of stimulus changes (Ritter et al. 1979; Näätänen 1982), the later component (N2b) is interpreted as a correlate of controlled detection of stimulus changes and phonological categorization (Ritter et al. 1979; Näätänen 1982; Amenedo and Díaz 1998; Näätänen et al. 2007).

In auditory oddball, $\mathrm{N} 1$ amplitude is strongly modulated by attentional context and interstimulus interval (ISI; Hillyard et al. 1973; Rosburg et al. 2008; May and Tiitinen 2010). During the process of falling asleep, N1 gradually declines in amplitude, possibly because of the decrease in the level of attention, and during REM sleep it is approximately $25-50 \%$ of its waking amplitude (Crowley and Colrain 2004). According to Hamm et al. (2013), this indexes the earliest cognitively influenced auditory neuronal event reliably measureable with EEG (Hamm et al. 2013).

The effects of aging on ERPs vary among studies. With respect to changes of the $\mathrm{N} 2$ component from childhood to adulthood, most studies showed a decrease in the N2 amplitude and latency (Johnstone et al. 1996; Mueller et al. 2008). Regarding N1 latency, only Iragui et al. (1993) reported significant age-related increases at $\mathrm{Cz}$, while the common finding has been that $\mathrm{N} 1$ latency remains unchanged with advancing age (Barrett et al. 1987). Ladish and Polich (1989) found an increase in N1 amplitude and a decrease in N1 latency with increasing age from 5 to 19 years, but other studies reported variations of $\mathrm{N} 1$ amplitude from childhood to adolescence (Ladish and Polich 1989; Ponton et al. 2000; Čeponienè et al. 2002, 2003, 2008). As for findings regarding adult development and aging, N1 amplitude increased significantly with age and increased N1 latency was only significant in the posterior region (Anderer et al. 1996).

This wide spectrum of results, findings and variety of auditory stimuli used, has possibly delayed the translation of N1 and N2 to clinical practice. Without linking auditory $\mathrm{N} 1$ and $\mathrm{N} 2$ to a restricted neuropsychological meaning, we are undoubtedly and objectively assessing the first stages of cognitive auditory processing.

\section{Aims and objectives}

The clinical significance and application of auditory ERPs such as $\mathrm{N} 1$ and $\mathrm{N} 2$ also, have remained marginal in the current practice of audiology, neurophysiology, neurology or psychiatry. Particularly the N1 wave, as an exogenous and robust auditory ERP may be used to assess central auditory processing.

This review will address the following aspects of $\mathrm{N} 1$ and N2: latency, amplitude, electrode location (most prominent $-\mathrm{Cz}$ and $\mathrm{Fz}$ ), development and maturation, aging, data published by year and country, type of stimuli in auditory oddball and research areas. We purpose that this review may lead to the first human normative values for $\mathrm{N} 1$ and N2 components when elicited by auditory oddball, being beneficial for a standard, controlled and wide accepted application to assess central auditory processing.

\section{Methods}

For this review, we searched EBSCO, PubMed, Web of Knowledge and Google Scholar to identify potential studies, without pre-defined time-window. Further studies were identified through references and citations. As inclusion criteria, we searched the term "N1" limited by the term "auditory oddball" in title, abstract, keyword or topic. Papers with no control group and animal research were excluded.

A search between ending November and early December 2013 found 2764 articles. Duplicates were removed and articles with no full text available were kindly asked by mail to authors, leaving a selection of papers for assessment of eligibility upon reading the full text article.

Data extraction, quality and relevance assessments

Data extracted from each full text article for eligibility assessment included: Authors; Year Publication; Aim; Country; Research areas; Control group $(N)$, age (mean); Type of stimuli (frequency, intensity, speech/consonantvowel, pure-tone); Electrodes recording for target/deviant stimuli (amplitude and latency of N1 and N2); Findings and suggestions. The complete systematic process of deduction can be found below in Fig. 1 .

Polynomial regression was applied as a descriptive method to data analysis that better fits a nonlinear model.

\section{Results}

A total of 2764 papers were initially identified in the database search from which 19 were hand searched and additional references. After, 2680 papers that did not meet inclusion criteria or duplicated were removed through title and abstract screening, leaving a total of 84 papers. Sixteen papers could not be accessed, 14 of which were not archived or we did not receive answer from authors and two were foreign language articles. A final total of 68 full text articles were assessed for eligibility, none of which required translation. Study's authors, country, mean age of control groups, number of participants, results for amplitude and latency across $\mathrm{Fz}$ and $\mathrm{Cz}$ electrodes for deviant 
Fig. 1 PRISMA systematic search flow diagram (Moher et al. 2009)

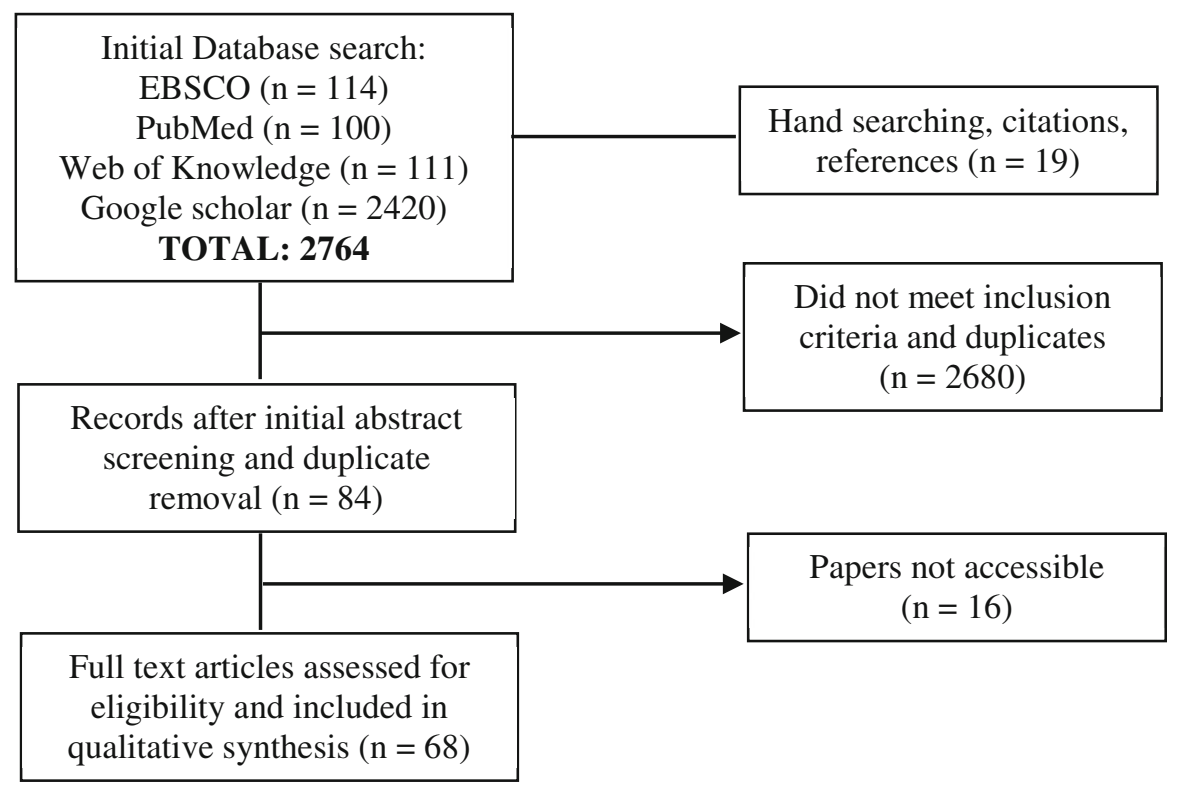

stimuli and subject's article (notes) are summarized in Tables 1 and 2, for N1 and N2, respectively. Many studies have results for different electrode locations. We considered $\mathrm{Fz}$ and $\mathrm{Cz}$ since they are the most common locations and were maximal amplitude for $\mathrm{N} 1, \mathrm{~N} 2$ and MMN is achieved (Näätänen and Picton 1987; Winkler 2007; Duncan et al. 2009; May and Tiitinen 2010). Studies with mean global field power were considered as $\mathrm{Cz}$ data, representing the standard deviation across electrodes at a given time point indicating the presence of a specific underlying neuronal component (Murray et al. 2008; Altieri 2013).

The results revealed papers between 1988 and 2013, last 25 years. The majority of the studies were published between 1995 and 1999, also it was when most of the studies elicited and analyzed both N1 and N2 (presented in Fig. 2). The five most published research areas were neurosciences/neurology, psychology, physiology, psychiatry and engineering. The five countries/territories with more papers published were USA, Australia, Germany, Japan and Austria.

Data analysis revealed a total of 3934 participants from control groups (Table 3), 2427 for N1 studies $($ male $=1247$, female $=1180)$, ranging in age from 6.6 to 85.0 years with a mean of $34.4(\mathrm{SD}=18.6)$. Regarding N2 studies, a total of 1507 participants were obtained from all control groups (male $=769$, female $=738$ ), ranging in age from 9.0 to 85.0 years with a mean of 36.1 $(\mathrm{SD}=20.7)$. All participants from control groups were reported to have normal hearing and vision, no neurological, psychiatric or other disorder. The majority of studies applied a frequency auditory oddball. The most standard stimuli used was a pure-tone of $1000 \mathrm{~Hz}$ at an intensity level range from 55 to $109 \mathrm{~dB}$ above hearing threshold, with at least $200 \mathrm{~Hz}$ of difference to the deviant stimuli, except in Demiralp et al. (1999), Wang et al. (2005) and Čeponiené et al. (2008) studies. Others used duration auditory oddball (Shelley et al. 1999; Segalowitz et al. 2001; Bortoletto et al. 2011; Wetzel et al. 2011; Neuhaus et al. 2013) and few intensity auditory oddball (Anderer et al. 1996, 1998a, b; Wang and Wang 2001; Wang et al. 2005; Barry et al. 2006). Only two papers elicited N1 and N2 with speech stimuli (Henkin et al. 2002; Toscano et al. 2010), due to the scarce data and type of stimuli they were not considered in the regression analysis.

Orthogonal polynomial regression equations were fit to the data, including degrees 4 through 6 to significantly increase predictability.

For $\mathrm{N} 1$, results revealed at $\mathrm{Cz}$ a mean amplitude of $7.3 \mu \mathrm{V}(\mathrm{SD}=3.0)$ ranging from 2.0 to $15.1 \mu \mathrm{V}$ (data from 69 cells) and a mean latency of $107.6 \mathrm{~ms}(\mathrm{SD}=15.0)$ ranging from 82.0 to $164.0 \mathrm{~ms}$ (data from 75 cells). At Fz, mean amplitude was $7.0 \mu \mathrm{V}(\mathrm{SD}=3.2)$ ranging from 1.1 to 14.4 (data from 48 cells) and a mean latency of $118.2 \mathrm{~ms}$ $(\mathrm{SD}=31.5)$ ranging from 88.9 to $260.0 \mathrm{~ms}$ (data from 45 cells).

Regarding N2, results revealed at $\mathrm{Cz}$ a mean amplitude of $4.9 \mu \mathrm{V}(\mathrm{SD}=2.6)$ ranging from 1.0 to $14.2 \mu \mathrm{V}$ (data from 40 cells) and a mean latency of $231.4 \mathrm{~ms}$ $(\mathrm{SD}=33.9)$ ranging from 200.0 to $371.0 \mathrm{~ms}$ (data from 43 cells). At $\mathrm{Fz}$ the mean amplitude was $5.7 \mu \mathrm{V}(\mathrm{SD}=3.9)$ ranging from 0 to $16.3 \mu \mathrm{V}$ (data from 34 cells) and a mean latency of $231.8 \mathrm{~ms}(\mathrm{SD}=18.9)$ ranging from 200.0 to $277.0 \mathrm{~ms}$ (data from 32 cells). Table 3 summarizes the results for $\mathrm{N} 1$ and $\mathrm{N} 2$ by four age ranges, suggested as normative values. 
Table 1 Studies with auditory oddball N1 elicitation (control groups data from deviant stimuli at Fz and Cz)

\begin{tabular}{|c|c|c|c|c|c|c|c|c|c|}
\hline References & Country & $N$ & Age & $\begin{array}{l}\mathrm{Cz} \\
(\mathrm{amp})\end{array}$ & $\begin{array}{l}\mathrm{Fz} \\
(\mathrm{amp})\end{array}$ & $\begin{array}{l}\mathrm{Cz} \\
\text { (lat) }\end{array}$ & $\begin{array}{l}\mathrm{Fz} \\
\text { (lat) }\end{array}$ & Stimuli & Notes \\
\hline Hegerl et al. (1988) & Germany & 27 & 34.1 & 12.2 & & 91.4 & & $\begin{array}{l}\text { Std: } 1600 \mathrm{~Hz}, \text { Dev: } \\
800 \mathrm{~Hz} / 65 \mathrm{~dB}\end{array}$ & Schizophrenia prognosis \\
\hline Verma et al. (1989) & USA & 11 & 63.5 & & & 103.7 & & $\begin{array}{l}\text { Std: } 1000 \mathrm{~Hz}, \text { Dev: } \\
2000 \mathrm{~Hz} / 60 \mathrm{~dB}\end{array}$ & Dementia \\
\hline \multirow[t]{3}{*}{ Iragui et al. (1993) } & \multirow[t]{3}{*}{ USA } & 28 & 29 & 6.6 & & 94 & & \multirow{3}{*}{$\begin{array}{l}\text { Std: } 1000 \mathrm{~Hz}, \text { Dev: } \\
1500 \mathrm{~Hz} / 70 \mathrm{~dB}\end{array}$} & \multirow{3}{*}{$\begin{array}{l}\text { Aging; correlations; } \\
\text { reaction time }\end{array}$} \\
\hline & & 16 & 49 & 8.1 & & 95 & & & \\
\hline & & 27 & 71 & 6.6 & & 97 & & & \\
\hline $\begin{array}{l}\text { Lembreghts et al. } \\
\text { (1995) }\end{array}$ & Belgium & 86 & 34.1 & 6.6 & 6.7 & 94 & 95 & $\begin{array}{l}\text { Std: } 800 \mathrm{~Hz}, \text { Dev: } 1470 \mathrm{~Hz} / \\
\quad 70 \mathrm{~dB}\end{array}$ & $\begin{array}{l}\text { Age, gender, } \\
\text { intervariability }\end{array}$ \\
\hline Tarter et al. (1995) & USA & 56 & 13.2 & 9.5 & & 119.9 & & $\begin{array}{l}\text { Std: } 1000 \mathrm{~Hz}, \text { Dev: } \\
2000 \mathrm{~Hz} / 64.5 \mathrm{~dB}\end{array}$ & $\begin{array}{l}\text { Adolescence; substance } \\
\text { abuse }\end{array}$ \\
\hline Winter et al. (1995) & Netherlands & 13 & 21.4 & 5.3 & 6.7 & 105 & 103 & $\begin{array}{l}\text { Std: } 1000 \mathrm{~Hz}, \text { Dev: } 1200 \\
\text { and } 2000 \mathrm{~Hz} / 65 \mathrm{~dB}\end{array}$ & Sleep stage 2 \\
\hline $\begin{array}{l}\text { Siedenberg et al. } \\
\text { (1996) }\end{array}$ & USA & 10 & 36.5 & & & 103 & & $\begin{array}{l}\text { Std: } 1000 \mathrm{~Hz}, \text { Dev: } \\
2000 \mathrm{~Hz} / 65 \mathrm{~dB}\end{array}$ & ERP vs ERF \\
\hline \multirow{7}{*}{$\begin{array}{l}\text { Anderer et al. (1996, } \\
\text { 1998a, b) }\end{array}$} & \multirow[t]{7}{*}{ Austria } & 58 & 25 & 8.6 & & 95 & & \multirow{7}{*}{$\begin{array}{l}\text { Std: } 90 \mathrm{~dB} \text { Dev: } 70 \mathrm{~dB} / \\
1000 \mathrm{~Hz}\end{array}$} & \multirow[t]{7}{*}{ Aging; ERPs; LORETA } \\
\hline & & 19 & 35 & 8.3 & & 100 & & & \\
\hline & & 13 & 45 & 8.7 & & 99 & & & \\
\hline & & 33 & 55 & 9.2 & & 95 & & & \\
\hline & & 29 & 65 & 9 & & 96 & & & \\
\hline & & 12 & 75 & 8.5 & & 93 & & & \\
\hline & & 8 & 85 & 8.1 & & 96 & & & \\
\hline Wright et al. (1996) & Australia & 28 & 62.8 & 13.6 & & 164 & & $\begin{array}{l}\text { Std: } 1000 \mathrm{~Hz}, \mathrm{Dev}: \\
2000 \mathrm{~Hz} / 60 \mathrm{~dB}+\text { back } \\
\text { noise }\end{array}$ & Parkinson's disease \\
\hline \multirow{5}{*}{$\begin{array}{l}\text { Johnstone et al. } \\
\text { (1996) }\end{array}$} & \multirow[t]{5}{*}{ Australia } & 10 & 9.1 & 9.6 & 12.1 & 132 & 133 & \multirow{5}{*}{$\begin{array}{l}\text { Std: } 1000 \mathrm{~Hz}, \text { Dev: } \\
1500 \mathrm{~Hz} / 60 \mathrm{~dB}\end{array}$} & \multirow{5}{*}{$\begin{array}{l}\text { Child and adolescent; } \\
\text { waves morphology; ERPs }\end{array}$} \\
\hline & & 10 & 11.2 & & & & & & \\
\hline & & 10 & 12.8 & 8.3 & 9.1 & 127 & 133 & & \\
\hline & & 10 & 15 & & & & & & \\
\hline & & 10 & 16.8 & 6.3 & 8.3 & 123 & 124 & & \\
\hline Hirata et al. (1996) & Japan & 14 & 66.8 & 6.9 & & 82 & & $\begin{array}{l}\text { Std: } 2000 \mathrm{~Hz}, \text { Dev: } \\
1000 \mathrm{~Hz} / 80 \mathrm{~dB}\end{array}$ & Stroke \\
\hline Akaho (1996) & Japan & 47 & 21.9 & 9.1 & & 100.2 & & $\begin{array}{l}\text { Std: } 1000 \mathrm{~Hz}, \mathrm{Dev}: \\
2000 \mathrm{~Hz} / 70 \mathrm{~dB}\end{array}$ & Cognition; effects of AED \\
\hline Kazis et al. (1996) & Greece & 53 & 45.6 & 10.3 & & 98.9 & & $\begin{array}{l}\text { Std: } 1000 \mathrm{~Hz}, \text { Dev: } \\
2000 \mathrm{~Hz} / 70 \mathrm{~dB}\end{array}$ & Myotonic dystrophy \\
\hline Brigham et al. (1997) & USA & 29 & 11.5 & 5.9 & 8.1 & 157.9 & 168.4 & $\begin{array}{l}\text { Std: } 1000 \mathrm{~Hz}, \mathrm{Dev}: \\
2000 \mathrm{~Hz} / 64.5 \mathrm{~dB}\end{array}$ & Polysubstance and alcohol \\
\hline Haig et al. (1997) & Australia & 25 & 27.3 & 8.6 & & 99 & & $\begin{array}{l}\text { Std: } 1000 \mathrm{~Hz}, \text { Dev: } \\
1500 \mathrm{~Hz} / 60 \mathrm{~dB}\end{array}$ & $\begin{array}{l}\text { Gaussian component; } \\
\text { schizophrenia }\end{array}$ \\
\hline Potts et al. (1998a) & USA & 24 & 39 & 3.8 & & 100 & & $\begin{array}{l}\text { Std: } 1000 \mathrm{~Hz}, \text { Dev: } \\
1500 \mathrm{~Hz} / 97 \mathrm{~dB}\end{array}$ & Schizophrenia \\
\hline Potts et al. (1998b) & USA & 20 & 21.1 & 2 & 6.7 & 100 & 110 & Std: $440 \mathrm{~Hz}$, Dev: $1245 \mathrm{~Hz}$ & Various ERPs \\
\hline \multirow{3}{*}{$\begin{array}{l}\text { Amenedo and Díaz } \\
\text { (1998) }\end{array}$} & \multirow[t]{3}{*}{ Spain } & 20 & 30.5 & 5.7 & 5.2 & 112 & 108 & \multirow{3}{*}{$\begin{array}{l}\text { Std: } 1000 \mathrm{~Hz}, \text { Dev: } \\
2000 \mathrm{~Hz} / 90 \mathrm{~dB}\end{array}$} & \multirow[t]{3}{*}{ Aging; ERPs } \\
\hline & & 20 & 50 & 7.5 & 7 & 105 & 104 & & \\
\hline & & 33 & 71.5 & 7.1 & 6.5 & 110 & 108 & & \\
\hline $\begin{array}{l}\text { Gonsalvez et al. } \\
\text { (1999) }\end{array}$ & Australia & 12 & 28.3 & 10.5 & 8.4 & 110 & 105 & $\begin{array}{l}\text { Std: } 1000 \mathrm{~Hz}, \text { Dev: } \\
1200 \mathrm{~Hz} / 60 \mathrm{~dB}\end{array}$ & $\begin{array}{l}\text { Target-to-target hypothesis } \\
\text { (P3) }\end{array}$ \\
\hline Shelley et al. (1999) & USA & 17 & 37.4 & & 8.0 & & 102.4 & $\begin{array}{l}\text { Std: } 1000 \mathrm{~Hz}, \text { Dev: } \\
1200 \mathrm{~Hz} / 75 \mathrm{~dB} \text { (ISI: } \\
\text { variable) }\end{array}$ & $\begin{array}{l}\text { Schizophrenia; cortical } \\
\text { dysfunction }\end{array}$ \\
\hline
\end{tabular}


Table 1 continued

\begin{tabular}{|c|c|c|c|c|c|c|c|c|c|}
\hline References & Country & $N$ & Age & $\begin{array}{l}\mathrm{Cz} \\
\text { (amp) }\end{array}$ & $\begin{array}{l}\mathrm{Fz} \\
\text { (amp) }\end{array}$ & $\begin{array}{l}\mathrm{Cz} \\
\text { (lat) }\end{array}$ & $\begin{array}{l}\mathrm{Fz} \\
\text { (lat) }\end{array}$ & Stimuli & Notes \\
\hline Demiralp et al. (1999) & Turkey & 10 & 21.5 & 11.6 & 10.2 & 125 & 125 & $\begin{array}{l}\text { Std: } 2000 \mathrm{~Hz}, \text { Dev: } \\
1950 \mathrm{~Hz} / 60 \mathrm{~dB}\end{array}$ & $\begin{array}{l}\text { Wavelet transform; } \\
\text { cognitive processes }\end{array}$ \\
\hline Gölgeli et al. (1999) & Turkey & 38 & 20.6 & 9.2 & 9 & 107 & 113 & $\begin{array}{l}\text { Std: } 2000 \mathrm{~Hz}, \text { Dev: } \\
1500 \mathrm{~Hz}\end{array}$ & Gender differences \\
\hline Barry et al. (2000) & Australia & 14 & 30.5 & 6 & & 117.6 & & $\begin{array}{l}\text { Std: } 1000 \mathrm{~Hz}, \mathrm{Dev}: \\
1500 \mathrm{~Hz} / 60 \mathrm{~dB}\end{array}$ & Alpha activity \\
\hline Sumi et al. (2000) & Japan & 39 & 68.5 & & $\mathrm{Pz}$ & & & $\begin{array}{l}\text { Std: } 2000 \mathrm{~Hz}, \text { Dev: } \\
1000 \mathrm{~Hz} / 70 \mathrm{~dB}\end{array}$ & Alzheimer \\
\hline Reinvang et al. (2000) & Norway & 27 & 29.7 & 6.4 & 4.9 & 99 & 97 & $\begin{array}{l}\text { Std: } 800 \mathrm{~Hz}, \text { Dev: } 1200 \mathrm{~Hz} / \\
\quad 80 \mathrm{~dB}\end{array}$ & Head injury \\
\hline $\begin{array}{l}\text { Johnstone et al. } \\
\text { (2001) }\end{array}$ & Australia & 50 & 12.5 & & 7.8 & & & $\begin{array}{l}\text { Std: } 1000 \mathrm{~Hz}, \text { Dev: } \\
1500 \mathrm{~Hz} / 60 \mathrm{~dB}\end{array}$ & Two sub types AD/HD \\
\hline Ford et al. (2001) & USA & 32 & 38 & 9.3 & 7.3 & 105 & 100 & $\begin{array}{l}\text { Std: } 500 \mathrm{~Hz}, \text { Dev: } 1000 \mathrm{~Hz} / \\
\quad 80 \mathrm{~dB}\end{array}$ & Epilepsy and schizophrenia \\
\hline $\begin{array}{l}\text { Segalowitz et al. } \\
\text { (2001) }\end{array}$ & Canada & 12 & 20.7 & & 8.2 & & 119.3 & $\begin{array}{l}\text { Std: } 800 \mathrm{~Hz} \text {, Dev: } 1500 \mathrm{~Hz} / \\
100 \mathrm{~ms}\end{array}$ & Mild head injury \\
\hline $\begin{array}{l}\text { Ullsperger et al. } \\
(2001)\end{array}$ & Germany & 15 & 23.8 & 4.5 & 6.3 & 133.3 & 133.3 & $\begin{array}{l}\text { Std: } 1000 \mathrm{~Hz}, \text { Dev: } \\
2000 \mathrm{~Hz} / 55 \mathrm{~dB}\end{array}$ & Mental workload changes \\
\hline $\begin{array}{l}\text { Wang and Wang } \\
(2001)\end{array}$ & China & 39 & 26.6 & 11.4 & 10.2 & 120.1 & 118.6 & $\begin{array}{l}\text { Std: } 0 \mathrm{~dB} \text {, Dev: } 60 \mathrm{~dB} / \\
2000 \mathrm{~Hz}\end{array}$ & Sensation seeking \\
\hline \multirow[t]{2}{*}{ Brown et al. (2002) } & \multirow[t]{2}{*}{ Australia } & 40 & 36.7 & 11.4 & 10.2 & 103.1 & 108.3 & \multirow{2}{*}{$\begin{array}{l}\text { Std: } 1000 \mathrm{~Hz}, \mathrm{Dev}: \\
1500 \mathrm{~Hz} / 60 \mathrm{~dB}\end{array}$} & \multirow[t]{2}{*}{ 1st episode schizophrenia } \\
\hline & & 40 & 19.7 & 10.6 & 9.3 & 104.5 & 110.4 & & \\
\hline Cohen et al. (2002) & USA & 39 & 24.7 & 4.9 & 5.5 & 117 & 117.6 & $\begin{array}{l}\text { Std: } 600 \mathrm{~Hz}, \text { Dev: } 1600 \mathrm{~Hz} / \\
\quad 60 \mathrm{~dB}\end{array}$ & Alcohol \\
\hline Henkin et al. (2002) & Israel & 20 & 14.4 & 8 & 7.5 & 103.9 & & $\begin{array}{l}\text { Std: } 1400 \mathrm{~Hz}, \mathrm{Dev}: \\
1000 \mathrm{~Hz}\end{array}$ & $\begin{array}{l}\text { AERPs; phonetic and } \\
\text { semantic processing }\end{array}$ \\
\hline $\begin{array}{l}\text { Valkonen-Korhonen } \\
\text { et al. (2003) }\end{array}$ & Finland & 19 & 29 & 5.0 & & 100 & & $\begin{array}{l}\text { Std: } 800 \mathrm{~Hz} \text {, Dev: } 560 \mathrm{~Hz} / \\
55 \mathrm{~dB}\end{array}$ & $\begin{array}{l}\text { Auditory processing; } \\
\text { psychotic }\end{array}$ \\
\hline Lucchesi et al. (2003) & Brazil & 12 & 24.8 & 10 & 11.2 & 100 & 100 & $\begin{array}{l}\text { Std: } 800 \mathrm{~Hz}, \text { Dev: } 1500 \mathrm{~Hz} / \\
\quad 70 \mathrm{~dB}\end{array}$ & Flunitrazepam effect \\
\hline Barry et al. (2003) & Australia & 16 & 28 & 6.3 & & 90 & & $\begin{array}{l}\text { Std: } 1000 \mathrm{~Hz}, \text { Dev: } \\
1500 \mathrm{~Hz} / 80 \mathrm{~dB}\end{array}$ & $\begin{array}{l}\text { EEG brain states; effects } \\
\text { ERPs }\end{array}$ \\
\hline Barry et al. (2004) & Australia & 14 & 31 & 8.7 & 9.6 & 114 & 114.5 & $\begin{array}{l}\text { Std: } 1000 \mathrm{~Hz}, \text { Dev: } \\
1500 \mathrm{~Hz} / 60 \mathrm{~dB}\end{array}$ & EEG alpha phase \\
\hline Mulert et al. (2004) & Germany & 9 & 24.2 & 10.2 & & 119.3 & & $\begin{array}{l}\text { Std: } 800 \mathrm{~Hz}, \text { Dev: } 1300 \mathrm{~Hz} / \\
95 \mathrm{~dB}\end{array}$ & fMRI; source localization \\
\hline Chao et al. (2004) & USA & 15 & 44.1 & 6.5 & & 90.9 & & $\begin{array}{l}\text { Std: } 1000 \mathrm{~Hz}, \text { Dev: } \\
2000 \mathrm{~Hz} / 55 \mathrm{~dB}\end{array}$ & Suppressed HIV patients \\
\hline \multirow[t]{3}{*}{ Wang et al. (2005) } & \multirow[t]{3}{*}{ USA } & 6 & 32.3 & & 1.1 & & 145 & \multirow{3}{*}{$\begin{array}{l}\text { Std: } 440 \mathrm{~Hz}, 80 \mathrm{~dB}, \mathrm{Dev}: \\
494 \mathrm{~Hz} 80 \mathrm{~dB} \text {, e } 440 \mathrm{~Hz} \\
65 \mathrm{~dB}\end{array}$} & \multirow{3}{*}{$\begin{array}{l}\text { TWI; development of } \\
\text { auditory processing; } \\
\text { MMN }\end{array}$} \\
\hline & & 9 & 10.8 & & 5.3 & & 222 & & \\
\hline & & 11 & 6.6 & & 5.6 & & 260 & & \\
\hline Gilmore et al. (2005) & USA & 14 & 43.6 & & 2.1 & 102 & & $\begin{array}{l}\text { Std: } 1000 \mathrm{~Hz}, \text { Dev: } \\
2000 \mathrm{~Hz} / 76 \mathrm{~dB}\end{array}$ & Schizophrenia \\
\hline Chunhau et al. (2005) & Japan & 16 & 23.5 & 7 & 7.7 & 101 & 109 & $\begin{array}{l}\text { Std: } 1000 \mathrm{~Hz}, \text { Dev: } \\
2000 \mathrm{~Hz} / 60 \mathrm{~dB}\end{array}$ & 40 min oddball \\
\hline Brown et al. (2006) & Australia & 20 & 24.8 & 2.7 & 3.7 & 105 & 105 & $\begin{array}{l}\text { Std: } 1000 \mathrm{~Hz}, \text { Dev: } \\
2000 \mathrm{~Hz} / 60 \mathrm{~dB}\end{array}$ & Inter-modal attention \\
\hline Barry et al. (2006) & Australia & 20 & 36 & 2.3 & 4.2 & 139 & 144 & $\begin{array}{l}\text { Std: } 50 \mathrm{~dB} \text {, Dev: } 80 \mathrm{~dB} / \\
1000 \mathrm{~Hz}\end{array}$ & $\begin{array}{l}\text { Narrow band EEG phase } \\
\text { effects }\end{array}$ \\
\hline $\begin{array}{l}\text { van Harten et al. } \\
\text { (2006) }\end{array}$ & Netherlands & 53 & 73.6 & 8.3 & 12 & 100.8 & 100.8 & $\begin{array}{l}\text { Std: } 1000 \mathrm{~Hz}, \text { Dev: } \\
2000 \mathrm{~Hz} / 80 \mathrm{~dB}\end{array}$ & $\begin{array}{l}\text { Vascular cognitive } \\
\text { impairment; peak-to-peak } \\
\text { ERPs }\end{array}$ \\
\hline
\end{tabular}


Table 1 continued

\begin{tabular}{|c|c|c|c|c|c|c|c|c|c|}
\hline References & Country & $N$ & Age & $\begin{array}{l}\mathrm{Cz} \\
\text { (amp) }\end{array}$ & $\begin{array}{l}\mathrm{Fz} \\
(\mathrm{amp})\end{array}$ & $\begin{array}{l}\mathrm{Cz} \\
\text { (lat) }\end{array}$ & $\begin{array}{l}\mathrm{Fz} \\
\text { (lat) }\end{array}$ & Stimuli & Notes \\
\hline Dixit et al. (2006) & India & 40 & 21.5 & 6.1 & 7.4 & 110.7 & 100.4 & $\begin{array}{l}\text { Std: } 1000 \mathrm{~Hz}, \text { Dev: } \\
2000 \mathrm{~Hz} / 60 \mathrm{~dB}\end{array}$ & Caffeine users \\
\hline \multirow{2}{*}{$\begin{array}{l}\text { Čeponienè et al. } \\
\text { (2008) }\end{array}$} & \multirow[t]{2}{*}{ USA } & 19 & 25.5 & 2.4 & 2.4 & 119 & & \multirow{2}{*}{$\begin{array}{l}\text { Std: } 500 \mathrm{~Hz}, \text { Dev: } 550 \mathrm{~Hz} / \\
\quad 63 \mathrm{~dB}\end{array}$} & \multirow[t]{2}{*}{ Aging; N1-N2-P2 } \\
\hline & & 19 & 71.3 & 2.1 & 2.0 & 118 & & & \\
\hline Kreukels et al. (2008) & Netherlands & 23 & 53.2 & 8.7 & & 101 & & $\begin{array}{l}\text { Std: } 1000 \mathrm{~Hz}, \text { Dev: } \\
2000 \mathrm{~Hz} / 75 \mathrm{~dB}\end{array}$ & $\begin{array}{l}\text { Chemotherapy; N1 } \\
\text { independent from P3 }\end{array}$ \\
\hline Zhu et al. (2008) & China & 15 & 23 & 4.9 & 7.7 & 110 & 120 & $\begin{array}{l}\text { Std: } 1000 \mathrm{~Hz}, \mathrm{Dev}: \\
2000 \mathrm{~Hz} / 80 \mathrm{~dB}\end{array}$ & $\begin{array}{l}\text { Music; mandarin lexical } \\
\text { tones }\end{array}$ \\
\hline Wise et al. (2009) & Australia & 98 & 35.6 & 7.7 & 7.3 & 113.8 & 111.7 & $\begin{array}{l}\text { Std: } 500 \mathrm{~Hz}, \text { Dev: } 1000 \mathrm{~Hz} / \\
\quad 75 \mathrm{~dB}\end{array}$ & Panic disorder \\
\hline Guney et al. (2009) & Turkey & 32 & 37.1 & 11.6 & 12.2 & 97.7 & 97.2 & $\begin{array}{l}\text { Std: } 1000 \mathrm{~Hz}, \text { Dev: } \\
2000 \mathrm{~Hz} / 80 \mathrm{~dB}\end{array}$ & Tobacco smokers \\
\hline $\begin{array}{l}\text { Dassanayake et al. } \\
\text { (2009) }\end{array}$ & Sri Lanka & 38 & 49 & & & 99.1 & & $\begin{array}{l}\text { Std: } 1000 \mathrm{~Hz} \text { Dev: } \\
2000 \mathrm{~Hz} / 75 \mathrm{~dB}\end{array}$ & Pesticides \\
\hline Gilmore et al. (2009) & USA & 12 & 25 & 3.2 & 2.6 & 101 & 107.8 & $\begin{array}{l}\text { Std: } 1000 \mathrm{~Hz}, \text { Dev: } \\
2000 \mathrm{~Hz} / 76 \mathrm{~dB}\end{array}$ & Hemispheric differences \\
\hline Ogawa et al. (2009) & Japan & 19 & 64.5 & 5.7 & & 85.9 & & $\begin{array}{l}\text { Std: } 1000 \mathrm{~Hz}, \mathrm{Dev}: \\
2000 \mathrm{~Hz} / 80 \mathrm{~dB}\end{array}$ & $\begin{array}{l}\text { Amyotrophic lateral } \\
\text { sclerosis }\end{array}$ \\
\hline $\begin{array}{l}\text { Cahn and Polich } \\
\text { (2009) }\end{array}$ & USA & 16 & 45.5 & 3.1 & 3.3 & 105 & 105 & $\begin{array}{l}\text { Std: } 500 \mathrm{~Hz}, \text { Dev: } 1000 \mathrm{~Hz} / \\
\quad 80 \mathrm{~dB}\end{array}$ & $\begin{array}{l}\text { Meditation; different } \\
\text { mental states }\end{array}$ \\
\hline $\begin{array}{l}\text { Sakamoto et al. } \\
\text { (2009) }\end{array}$ & Japan & 11 & 30.9 & 5.5 & 6.7 & & & $\begin{array}{l}\text { Std: } 1000 \mathrm{~Hz}, \text { Dev: } \\
2000 \mathrm{~Hz} / 55 \mathrm{~dB}\end{array}$ & Mastication \\
\hline Whelan et al. (2010) & Ireland & 21 & 40.3 & & 1.6 & & 90.6 & Std: $500 \mathrm{~Hz}$, Dev: $1000 \mathrm{~Hz}$ & Multiple sclerosis \\
\hline Boucher et al. (2010) & Canada & 99 & 11.3 & 4.7 & & 127.1 & & $\begin{array}{l}\text { Std: } 1000 \mathrm{~Hz}, \text { Dev: } \\
2000 \mathrm{~Hz} / 70 \mathrm{~dB}\end{array}$ & $\begin{array}{l}\text { MeHg; PCBs; } \\
\text { neurodevelopment }\end{array}$ \\
\hline $\begin{array}{l}\text { Gandelman-Marton } \\
\text { et al. (2010) }\end{array}$ & Israel & 18 & 35.6 & & & 113.2 & 113.4 & $\begin{array}{l}\text { Std: } 1000 \mathrm{~Hz}, \text { Dev: } \\
2000 \mathrm{~Hz} / 70 \mathrm{~dB}\end{array}$ & $\begin{array}{l}\text { Immediate and short-term } \\
\text { retest }\end{array}$ \\
\hline $\begin{array}{l}\text { Bortoletto et al. } \\
\text { (2011) }\end{array}$ & Italy & 20 & 25 & 5.1 & & 133 & & $\begin{array}{l}5 \text { pair of } \mathrm{Hz} \text { and } 3 \text { different } \\
\text { ISI }\end{array}$ & Sleep deprivation \\
\hline Wetzel et al. (2011) & Germany & 18 & 25 & 4.5 & 2.2 & 110 & 110 & $\begin{array}{l}\text { Std: } 500 \mathrm{~Hz} \text {, Dev: } 200 \text { and } \\
500 \mathrm{~ms}\end{array}$ & $\begin{array}{l}\text { Novel; duration; better in } \\
\text { children }\end{array}$ \\
\hline \multirow[t]{2}{*}{ Tsai et al. (2012) } & \multirow[t]{2}{*}{ Taiwan } & 63 & 9 & & $\mathrm{Pz}$ & & & \multirow{2}{*}{$\begin{array}{l}\text { Std: } 3000 \mathrm{~Hz}, \text { Dev: } \\
2000 \mathrm{~Hz} / 60 \mathrm{~dB}\end{array}$} & \multirow{2}{*}{$\begin{array}{l}\text { ADHD; age-related; } \\
\text { correlations; }\end{array}$} \\
\hline & & 51 & 9 & 13.6 & 14.4 & 119.5 & 126.2 & & \\
\hline Tanaka et al. (2012) & Japan & 14 & 43 & 4.8 & & 99 & & $\begin{array}{l}\text { Std: } 1000 \mathrm{~Hz}, \mathrm{Dev}: \\
2000 \mathrm{~Hz} / 80 \mathrm{~dB}\end{array}$ & Myotonic dystrophy type 1 \\
\hline \multirow[t]{2}{*}{ Ho et al. (2012) } & \multirow[t]{2}{*}{ Taiwan } & 15 & 23.7 & & & 91 & 88.9 & \multirow{2}{*}{$\begin{array}{l}\text { Std: } 2000 \mathrm{~Hz} \text { Dev: } \\
1000 \mathrm{~Hz} / 80 \mathrm{~dB}\end{array}$} & \multirow[t]{2}{*}{ Normal aging } \\
\hline & & 15 & 70.1 & & & 95 & 90 & & \\
\hline Neuhaus et al. (2013) & Germany & 144 & 32.4 & 15.1 & 11.4 & 111.1 & 127.8 & $\begin{array}{l}\text { Std: } 500 \mathrm{~ms} 109 \mathrm{~dB} \text {, Dev: } \\
40 \mathrm{~ms} 83 \mathrm{~dB}\end{array}$ & $\begin{array}{l}\text { Schizophrenia; gatings; } \\
\text { AUC e ROC }\end{array}$ \\
\hline $\begin{array}{l}\text { Schneider et al. } \\
\text { (2013) }\end{array}$ & USA & 40 & 13.9 & 3.4 & & 125 & & $\begin{array}{l}\text { Std: } 1000 \mathrm{~Hz}, \text { Dev: } \\
2000 \mathrm{~Hz} / 70 \mathrm{~dB}\end{array}$ & $\begin{array}{l}\text { Fragile } X \text { syndrome } \\
\text { treatment }\end{array}$ \\
\hline Hamm et al. (2013) & USA & 70 & 38.4 & 2.2 & 2.2 & 92 & 92 & $\begin{array}{l}\text { Std: } 1500 \mathrm{~Hz}, \text { Dev: } \\
1000 \mathrm{~Hz} / 75 \mathrm{~dB}\end{array}$ & Bipolar disorder \\
\hline
\end{tabular}

Polynomial regression curves showed that latency of N1 component slightly decreases with aging at $\mathrm{Cz}(r=0.50$; Fig. 3) and Fz ( $r=0.78$; Fig. 4). As for amplitude, N1 seems to decrease from birth until 30 years and stabilize after 40 years old at $\mathrm{Cz}(r=0.20)$, but at $\mathrm{Fz}$ a similar decrement only finishes by 60 years and starts to highly increase after $(r=0.35)$. Regarding $\mathrm{N} 2$ component (Figs. 5, 6), latency did not covary with age for both $\mathrm{Cz}$ $(r=0.36)$ and $\mathrm{Fz}(r=0.44)$ locations. In contrast, N2 amplitude at $\mathrm{Cz}(r=0.68)$ and $\mathrm{Fz}(r=0.81)$ showed a significant decrement with age, with a curious slight increment between 20 and 45 years at $\mathrm{Cz}$. 
Table 2 Studies with auditory oddball N2 elicitation (control groups data from deviant stimuli at Fz and $\mathrm{Cz}$ )

\begin{tabular}{|c|c|c|c|c|c|c|c|c|c|}
\hline References & Country & $N$ & Age & $\begin{array}{l}\mathrm{Cz} \\
\text { (amp) }\end{array}$ & $\begin{array}{l}\mathrm{Fz} \\
\text { (amp) }\end{array}$ & $\begin{array}{l}\mathrm{Cz} \\
\text { (lat) }\end{array}$ & $\begin{array}{l}\mathrm{Fz} \\
\text { (lat) }\end{array}$ & Stimuli & Notes \\
\hline Verma et al. (1989) & USA & 11 & 63.5 & & & 237.8 & & $\begin{array}{l}\text { Std: } 1000 \mathrm{~Hz}, \text { Dev: } \\
2000 \mathrm{~Hz} / 60 \mathrm{~dB}\end{array}$ & Dementia \\
\hline \multirow[t]{3}{*}{ Iragui et al. (1993) } & \multirow[t]{3}{*}{ USA } & 28 & 29 & 2.26 & & 211 & & \multirow{3}{*}{$\begin{array}{l}\text { Std: } 1000 \mathrm{~Hz}, \text { Dev: } \\
1500 \mathrm{~Hz} / 70 \mathrm{~dB}\end{array}$} & \multirow{3}{*}{$\begin{array}{l}\text { Aging; correlations; reaction } \\
\text { time }\end{array}$} \\
\hline & & 16 & 49 & 3.9 & & 220 & & & \\
\hline & & 27 & 71 & 5.5 & & 231 & & & \\
\hline $\begin{array}{l}\text { Lembreghts et al. } \\
\text { (1995) }\end{array}$ & Belgium & 41 & 34.1 & 4.7 & 3.6 & 207 & 212 & $\begin{array}{l}\text { Std: } 800 \mathrm{~Hz}, \mathrm{Dev}: \\
1470 \mathrm{~Hz} / 70 \mathrm{~dB}\end{array}$ & Age, gender, intervariability \\
\hline \multirow[t]{7}{*}{ Anderer et al. (1996) } & \multirow[t]{7}{*}{ Austria } & 58 & 25 & 6.7 & 6.5 & 210 & 212 & \multirow{7}{*}{$\begin{array}{l}\text { Std: } 90 \mathrm{~dB} \text {, Dev: } 70 \mathrm{~dB} / \\
1000 \mathrm{~Hz}\end{array}$} & \multirow[t]{7}{*}{ Aging; ERPs } \\
\hline & & 19 & 35 & 5.6 & 4 & 213 & 218 & & \\
\hline & & 13 & 45 & 7.1 & 5 & 224 & 225 & & \\
\hline & & 33 & 55 & 8.1 & 3.8 & 221 & 220 & & \\
\hline & & 29 & 65 & 4.2 & 1.5 & 222 & 217 & & \\
\hline & & 12 & 75 & 3.1 & 1.9 & 212 & 212 & & \\
\hline & & 8 & 85 & 2 & 0 & 254 & 253 & & \\
\hline $\begin{array}{l}\text { Siedenberg et al. } \\
\text { (1996) }\end{array}$ & USA & 10 & 36.5 & & & 216 & & $\begin{array}{l}\text { Std: } 1000 \mathrm{~Hz}, \text { Dev: } \\
2000 \mathrm{~Hz} / 65 \mathrm{~dB}\end{array}$ & ERP vs ERF \\
\hline \multirow{5}{*}{$\begin{array}{l}\text { Johnstone et al. } \\
\text { (1996) }\end{array}$} & \multirow[t]{5}{*}{ Australia } & 10 & 9.1 & & 14.6 & & 253.3 & \multirow{5}{*}{$\begin{array}{l}\text { Std: } 1000 \mathrm{~Hz}, \text { Dev: } \\
1500 \mathrm{~Hz} / 60 \mathrm{~dB}\end{array}$} & \multirow{5}{*}{$\begin{array}{l}\text { ERPs; child and adolescent; } \\
\text { morphology }\end{array}$} \\
\hline & & 10 & 11.2 & & & & & & \\
\hline & & 10 & 12.8 & & & & & & \\
\hline & & 10 & 15 & 6.7 & 12.9 & 240 & 253.3 & & \\
\hline & & 10 & 16.8 & & 5.8 & & 253.3 & & \\
\hline Akaho (1996) & Japan & 47 & 21.9 & 3.5 & & 223.2 & & $\begin{array}{l}\text { Std: } 1000 \mathrm{~Hz}, \text { Dev: } \\
2000 \mathrm{~Hz} / 70 \mathrm{~dB}\end{array}$ & Cognition; effects of AED \\
\hline Kazis et al. (1996) & Greece & 53 & 45.6 & 8.4 & & & & $\begin{array}{l}\text { Std: } 1000 \mathrm{~Hz}, \text { Dev: } \\
2000 \mathrm{~Hz} / 70 \mathrm{~dB}\end{array}$ & Myotonic dystrophy \\
\hline Brigham et al. (1997) & USA & 29 & 11.5 & 7.3 & 16.3 & 221 & 221.1 & $\begin{array}{l}\text { Std: } 1000 \mathrm{~Hz}, \text { Dev: } \\
2000 \mathrm{~Hz} / 64.5 \mathrm{~dB}\end{array}$ & Polysubstance and alcohol \\
\hline Haig et al. (1997) & Australia & 25 & 27.3 & 4 & & 201 & & $\begin{array}{l}\text { Std: } 1000 \mathrm{~Hz}, \text { Dev: } \\
1500 \mathrm{~Hz} / 60 \mathrm{~dB}\end{array}$ & $\begin{array}{l}\text { Gaussian component; } \\
\text { schizophrenia }\end{array}$ \\
\hline \multirow{3}{*}{$\begin{array}{l}\text { Amenedo and Díaz } \\
\text { (1998) }\end{array}$} & \multirow[t]{3}{*}{ Spain } & 20 & 30.5 & 2.5 & 4.5 & 237 & 242 & \multirow{3}{*}{$\begin{array}{l}\text { Std: } 1000 \mathrm{~Hz}, \text { Dev: } \\
2000 \mathrm{~Hz} / 90 \mathrm{~dB}\end{array}$} & \multirow[t]{3}{*}{ Age-related ERPs } \\
\hline & & 20 & 50 & 3.4 & 3.1 & 250 & 252 & & \\
\hline & & 33 & 71.5 & 3.8 & 3.7 & 271 & 277 & & \\
\hline $\begin{array}{l}\text { Demiralp et al. } \\
\text { (1999) }\end{array}$ & Turkey & 10 & 21.5 & 6.8 & 3.4 & 270 & 270 & $\begin{array}{l}\text { Std: } 2000 \mathrm{~Hz}, \text { Dev: } \\
1950 \mathrm{~Hz} / 60 \mathrm{~dB}\end{array}$ & $\begin{array}{l}\text { Wavelet transform; cognitive } \\
\text { processes }\end{array}$ \\
\hline Gölgeli et al. (1999) & Turkey & 38 & 20.6 & 7.1 & 8 & 225 & 240 & $\begin{array}{l}\text { Std: } 2000 \mathrm{~Hz}, \text { Dev: } \\
1500 \mathrm{~Hz}\end{array}$ & Gender differences \\
\hline Sumi et al. (2000) & Japan & 39 & 68.5 & & $\mathrm{Pz}$ & & & $\begin{array}{l}\text { Std: } 2000 \mathrm{~Hz}, \text { Dev: } \\
1000 \mathrm{~Hz} / 70 \mathrm{~dB}\end{array}$ & Alzheimer \\
\hline $\begin{array}{l}\text { Reinvang et al. } \\
\text { (2000) }\end{array}$ & Norway & 27 & 29.7 & 2 & 1.5 & 207 & 207 & $\begin{array}{l}\text { Std: } 800 \mathrm{~Hz}, \mathrm{Dev}: \\
1200 \mathrm{~Hz} / 80 \mathrm{~dB}\end{array}$ & Head injury \\
\hline $\begin{array}{l}\text { Johnstone et al. } \\
\text { (2001) }\end{array}$ & Australia & 50 & 12.5 & & 8.5 & & 238.5 & $\begin{array}{l}\text { Std: } 1000 \mathrm{~Hz}, \text { Dev: } \\
1500 \mathrm{~Hz} / 60 \mathrm{~dB}\end{array}$ & Two sub types AD/HD \\
\hline $\begin{array}{l}\text { Segalowitz et al. } \\
\text { (2001) }\end{array}$ & Canada & 12 & 20.7 & & & 235.3 & & $\begin{array}{l}\text { Std: } 800 \mathrm{~Hz}, \mathrm{Dev}: \\
1500 \mathrm{~Hz} / 100 \mathrm{~ms}\end{array}$ & Mild head injury \\
\hline $\begin{array}{l}\text { Wang and Wang } \\
\text { (2001) }\end{array}$ & China & 39 & 26.6 & & 2.9 & 245.1 & 236.8 & $\begin{array}{l}\text { Std: } 0 \mathrm{~dB} \text {, Dev: } 60 \mathrm{~dB} / \\
2000 \mathrm{~Hz}\end{array}$ & Sensation seeking \\
\hline \multirow[t]{2}{*}{ Brown et al. (2002) } & \multirow[t]{2}{*}{ Australia } & 40 & 36.7 & 7.5 & 5.3 & 207.2 & 209.8 & \multirow{2}{*}{$\begin{array}{l}\text { Std: } 1000 \mathrm{~Hz} \text { Dev: } \\
1500 \mathrm{~Hz} / 60 \mathrm{~dB}\end{array}$} & \multirow[t]{2}{*}{ 1st episode Schizophrenia } \\
\hline & & 40 & 19.7 & 3.2 & 9.9 & 209.3 & 214.1 & & \\
\hline Cohen et al. (2002) & USA & 39 & 24.7 & 2.3 & 5.1 & 217.6 & 235.3 & $\begin{array}{l}\text { Std: } 600 \mathrm{~Hz}, \text { Dev: } \\
1600 \mathrm{~Hz} / 60 \mathrm{~dB}\end{array}$ & Alcohol \\
\hline
\end{tabular}


Table 2 continued

\begin{tabular}{|c|c|c|c|c|c|c|c|c|c|}
\hline References & Country & $N$ & Age & $\begin{array}{l}\mathrm{Cz} \\
\text { (amp) }\end{array}$ & $\begin{array}{l}\mathrm{Fz} \\
(\mathrm{amp})\end{array}$ & $\begin{array}{l}\mathrm{Cz} \\
\text { (lat) }\end{array}$ & $\begin{array}{l}\mathrm{Fz} \\
\text { (lat) }\end{array}$ & Stimuli & Notes \\
\hline Henkin et al. (2002) & Israel & 20 & 14.4 & 4.9 & 8.8 & & 246.3 & $\begin{array}{l}\text { Std: } 1400 \mathrm{~Hz}, \text { Dev: } \\
1000 \mathrm{~Hz}\end{array}$ & $\begin{array}{l}\text { AERPs; phonetic processing in } \\
\text { children }\end{array}$ \\
\hline $\begin{array}{l}\text { Valkonen-Korhonen } \\
\text { et al. (2003) }\end{array}$ & Finland & 19 & 29 & 2.4 & & 200 & & $\begin{array}{l}\text { Std: } 800 \mathrm{~Hz} \text {, Dev: } \\
560 \mathrm{~Hz} / 55 \mathrm{~dB}\end{array}$ & Auditory processing; psychotic \\
\hline Lucchesi et al. (2003) & Brazil & 12 & 24.8 & 5 & 3 & 200 & 200 & $\begin{array}{l}\text { Std: } 800 \mathrm{~Hz}, \text { Dev: } \\
1500 \mathrm{~Hz} / 70 \mathrm{~dB}\end{array}$ & Flunitrazepam effect; N2b \\
\hline $\begin{array}{l}\text { van Harten et al. } \\
\text { (2006) }\end{array}$ & Netherlands & 53 & 73.6 & 5.6 & 5.6 & 232.5 & 232.5 & $\begin{array}{l}\text { Std: } 1000 \mathrm{~Hz}, \mathrm{Dev}: \\
2000 \mathrm{~Hz} / 80 \mathrm{~dB}\end{array}$ & $\begin{array}{l}\text { Vascular cognitive impairment; } \\
\text { peak-to-peak ERPs }\end{array}$ \\
\hline Dixit et al. (2006) & India & 40 & 21.5 & 3.9 & 5.5 & 209.6 & 215.3 & $\begin{array}{l}\text { Std: } 1000 \mathrm{~Hz}, \text { Dev: } \\
2000 \mathrm{~Hz} / 60 \mathrm{~dB}\end{array}$ & Caffeine \\
\hline \multirow{2}{*}{$\begin{array}{l}\text { Čeponienė et al. } \\
(2008)\end{array}$} & \multirow[t]{2}{*}{ USA } & 19 & 25.5 & 2.5 & 2.4 & 341 & & \multirow{2}{*}{$\begin{array}{l}\text { Std: } 500 \mathrm{~Hz}, \mathrm{Dev}: \\
550 \mathrm{~Hz} / 63 \mathrm{~dB}\end{array}$} & \multirow[t]{2}{*}{ Aging; N1-N2-P2 } \\
\hline & & 19 & 71.3 & 1.0 & 1.1 & 371 & & & \\
\hline Zhu et al. (2008) & China & 15 & 23 & & 3.1 & & 225 & $\begin{array}{l}\text { Std: } 1000 \mathrm{~Hz}, \text { Dev: } \\
2000 \mathrm{~Hz} / 80 \mathrm{~dB}\end{array}$ & Music; mandarin lexical tones \\
\hline Wise et al. (2009) & Australia & 98 & 35.6 & 5.5 & 4.9 & 231.6 & 238 & $\begin{array}{l}\text { Std: } 500 \mathrm{~Hz}, \text { Dev: } \\
1000 \mathrm{~Hz} / 75 \mathrm{~dB}\end{array}$ & Panic disorder \\
\hline Guney et al. (2009) & Turkey & 32 & 37.1 & 8 & 10.0 & 216.3 & 225.8 & $\begin{array}{l}\text { Std: } 1000 \mathrm{~Hz}, \text { Dev: } \\
2000 \mathrm{~Hz} / 80 \mathrm{~dB}\end{array}$ & Tobacco smokers \\
\hline $\begin{array}{l}\text { Dassanayake et al. } \\
\text { (2009) }\end{array}$ & Sri Lanka & 38 & 49 & & & 226.3 & & $\begin{array}{l}\text { Std: } 1000 \mathrm{~Hz}, \text { Dev: } \\
2000 \mathrm{~Hz} / 75 \mathrm{~dB}\end{array}$ & Pesticides \\
\hline Ogawa et al. (2009) & Japan & 19 & 64.5 & 4.7 & & 210 & & $\begin{array}{l}\text { Std: } 1000 \mathrm{~Hz}, \text { Dev: } \\
2000 \mathrm{~Hz} / 80 \mathrm{~dB}\end{array}$ & Amyotrophic lateral sclerosis \\
\hline Whelan et al. (2010) & Ireland & 21 & 40.3 & 1.7 & & 211.7 & & $\begin{array}{l}\text { Std: } 500 \mathrm{~Hz}, \text { Dev: } \\
1000 \mathrm{~Hz}\end{array}$ & Multiple sclerosis \\
\hline $\begin{array}{l}\text { Gandelman-Marton } \\
\text { et al. (2010) }\end{array}$ & Israel & 18 & 35.6 & 7.1 & 7.1 & 221.2 & 223.1 & $\begin{array}{l}\text { Std: } 1000 \mathrm{~Hz}, \text { Dev: } \\
2000 \mathrm{~Hz} / 70 \mathrm{~dB}\end{array}$ & Immediate and short-term retest \\
\hline \multirow[t]{2}{*}{ Tsai et al. (2012) } & \multirow[t]{2}{*}{ Taiwan } & 63 & 9 & & $\mathrm{Pz}$ & & & \multirow{2}{*}{$\begin{array}{l}\text { Std: } 3000 \mathrm{~Hz}, \text { Dev: } \\
2000 \mathrm{~Hz} / 60 \mathrm{~dB}\end{array}$} & \multirow[t]{2}{*}{ Age-related; correlations ADHD } \\
\hline & & 51 & 9 & 14.2 & 10.8 & 240.7 & 239.2 & & \\
\hline Tanaka et al. (2012) & Japan & 14 & 43 & 5.9 & & 215 & & $\begin{array}{l}\text { Std: } 1000 \mathrm{~Hz}, \text { Dev: } \\
2000 \mathrm{~Hz} / 80 \mathrm{~dB}\end{array}$ & Myotonic dystrophy type 1 \\
\hline $\begin{array}{l}\text { Schneider et al. } \\
\text { (2013) }\end{array}$ & USA & 40 & 13.9 & 1.6 & & 285 & & $\begin{array}{l}\text { Std: } 1000 \mathrm{~Hz}, \text { Dev: } \\
2000 \mathrm{~Hz} / 70 \mathrm{~dB}\end{array}$ & Fragile $\mathrm{X}$ syndrome treatment \\
\hline
\end{tabular}

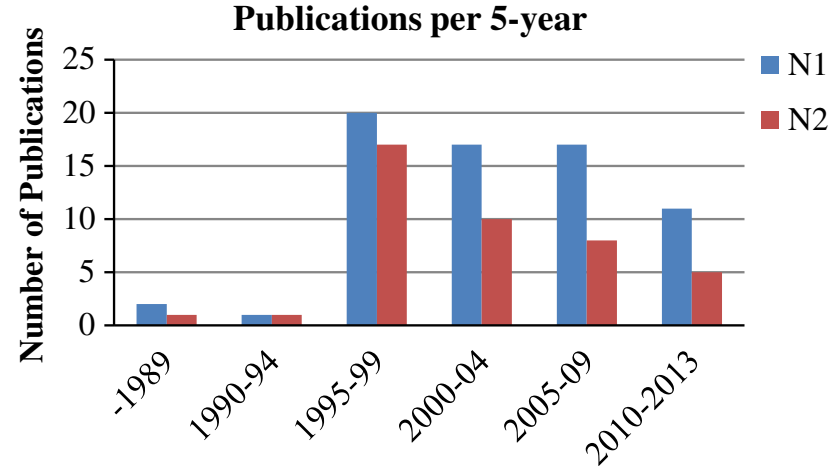

Fig. 2 Number of publications per 5-year for auditory oddball N1 (N2 included in N1 studies)

\section{Discussion}

Most of the studies assessed have elicited N1 and several $\mathrm{N} 2$ as well, in clinical groups, mainly in Neurology,
Psychiatry and Psychology areas. This fact, suggests that in the last decades N1 and N2 components have been studied as possible endophenotypes or bio-markers for different disorders and in some cases for recovery index: namely, in schizophrenia (Hegerl et al. 1988; Haig et al. 1997; Potts et al. 1998a; Shelley et al. 1999; Ford et al. 2001; Brown et al. 2002; Gilmore et al. 2005; Neuhaus et al. 2013), dementia (Verma et al. 1989), Alzheimer (Sumi et al. 2000), epilepsy and AED effects (Akaho 1996; Ford et al. 2001; Lucchesi et al. 2003), alcohol (Brigham et al. 1997; Cohen et al. 2002) and substance abuse (Tarter et al. 1995; Brigham et al. 1997), psychosis (Valkonen-Korhonen et al. 2003), panic disorder (Wise et al. 2009), Parkinson's disease (Wright et al. (1996), ADHD (Johnstone et al. 2001; Tsai et al. 2012), stroke (Hirata et al. 1996) and vascular cognitive impairment (van Harten et al. 2006), myotonic dystrophy (Kazis et al. 1996; Tanaka et al. 2012), head injury (Reinvang et al. 2000; Segalowitz et al. 2001), 
Table 3 Suggested normative values for N1 and N2 by age (control groups data from deviant stimuli at Fz and Cz)

\begin{tabular}{|c|c|c|c|c|c|c|c|}
\hline \multirow[t]{2}{*}{ Component } & \multirow[t]{2}{*}{ Age (years) } & \multirow[t]{2}{*}{$N(3934)$} & \multirow[t]{2}{*}{ Participants age (mean/SD) } & \multicolumn{2}{|c|}{ Amplitude $($ mean/SD) $(\mu \mathrm{V})$} & \multicolumn{2}{|c|}{ Latency $(\mathrm{mean} / \mathrm{SD})(\mathrm{ms})$} \\
\hline & & & & $\mathrm{Cz}$ & $\mathrm{Fz}$ & $\mathrm{Cz}$ & $\mathrm{Fz}$ \\
\hline \multirow[t]{4}{*}{ N1 } & $(6-20)$ & 518 & $12.3 / 3.3$ & $8.0 / 3.0$ & $8.8 / 2.8$ & $124.0 / 15.1$ & $160.0 / 54.0$ \\
\hline & $(21-40)$ & 1326 & $28.8 / 5.7$ & $7.1 / 3.2$ & $6.9 / 2.9$ & $107.2 / 11.8$ & $111.0 / 13.6$ \\
\hline & $(41-60)$ & 276 & $47.0 / 4.4$ & $7.4 / 2.3$ & $3.5 / 2.4$ & $99.1 / 4.3$ & $99.9 / 8.1$ \\
\hline & $(61-85)$ & 307 & $69.9 / 6.0$ & $7.6 / 2.9$ & $6.8 / 5.0$ & $103.5 / 21.4$ & $99.6 / 9.1$ \\
\hline \multirow[t]{4}{*}{$\mathrm{N} 2$} & $(9-20)$ & 343 & $12.9 / 3.3$ & $6.3 / 4.4$ & $11.0 / 3.5$ & $239.2 / 28.8$ & $239.9 / 15.1$ \\
\hline & $(21-40)$ & 706 & $28.3 / 5.8$ & $4.7 / 2.1$ & $4.7 / 2.2$ & $224.8 / 30.9$ & $225.6 / 17.5$ \\
\hline & $(41-60)$ & 208 & $47.1 / 4.6$ & $5.5 / 2.6$ & $4.0 / 1.0$ & $224.0 / 12.5$ & $232.3 / 17.2$ \\
\hline & $(61-85)$ & 250 & $70.9 / 6.3$ & $3.7 / 1.6$ & $2.3 / 2.0$ & $249.0 / 49.7$ & $238.3 / 26.9$ \\
\hline
\end{tabular}

suppressed HIV patients (Chao et al. 2004), chemotherapy (Kreukels et al. 2008) and pesticides exposure (Dassanayake et al. 2009), smoking (Guney et al. 2009), amyotrophic lateral sclerosis (Ogawa et al. 2009) and multiple sclerosis (Whelan et al. 2010), more recently fragile X syndrome treatment (Schneider et al. 2013), and bipolar disorder (Hamm et al. 2013).

In several respects, our data replicate the findings of others with regard to the effects of age on the amplitude and latency of N1 and N2. Regarding N1 latency, only Iragui et al. (1993) reported significant age-related increases at $\mathrm{Cz}$. The common finding has been that $\mathrm{N} 1$ latency remains unchanged or slightly decreases with advancing age for target stimuli at this electrode, from where it has usually been recorded (Goodin et al. 1978; Brown et al. 1983; Picton et al. 1984; Barrett et al. 1987; Anderer et al. 1996; Amenedo and Díaz 1998; Tsai et al. 2012). Further, Anderer et al. (1996, 1998a) found a latency increase over age only for standard stimuli. But at $\mathrm{Fz}, \mathrm{N} 1$ latency decrement is particularly pronounced during childhood and adolescence, this result confirms the assumptions that the maturation of the frontal N1 is not yet completed at the age of 9-12 years due to incomplete frontal myelination (Bruneau et al. 1997). Overall, the decrease in N1 latencies probably result from an increase in neural transmission speed due to age-related changes in myelination of underlying neural generators as well as increases in synaptic synchronization and efficacy (Huttenlocher 1979; Eggermont 1988; Courchesne 1990; Ponton et al. 1999).

A paradoxical but astonishing phenomenon seems to take place from childhood to adulthood. In 1979, Huttenlocher observed that a decline in synaptic density between ages 2 and 16 years was accompanied by a slight decrease in neuronal density. This is consistent with our results if we consider that synaptic density is related to N1 and $\mathrm{N} 2$ amplitudes. Both components have an amplitude decrement at $\mathrm{Fz}$ and $\mathrm{Cz}$ (Figs. 3, 4, 5, 6). Human cerebral cortex is one of a number of neuronal systems in which loss of neurons and synapses appears to occur as a late developmental event. The relationship of N1/N2 to the adult N1/ $\mathrm{N} 2$ is unclear. Maturational changes in the central auditory system are complex and extend well into the second decade of life (Sharma et al. 1997; Gilley et al. 2005). We believe that the effects of age, stimulation rate and education influence components morphology and amplitude, the latter is usually taken as an exclusion or inclusion criteria. The scarce literature on possible education effects on $\mathrm{N} 1$ and $\mathrm{N} 2$ does not enable us to achieve further conclusions. However, a profound and comprehensive revision on these topics should be made in a future article. In a recent systematic review and meta-analysis regarding P3, no education effects were found (van Dinteren et al. 2014). This was possible because $\mathrm{P} 3$ is one of the most studied components with over 12,000 publications in 50 years of intensive research (van Dinteren et al. 2014). Despite the interesting literature review and discussion made by van Dinteren et al. (2014), we cannot compare results since they analyzed data only for the $\mathrm{Pz}$ electrode site. Although, it definitely seems that N1/N2 and P3 have different development across the lifespan, due to different generators and underlying psychophysiological processes.

The range age between 41 and 60 years is the one with fewer participants, both to $\mathrm{N} 1$ and N2 (Table 3). This seems to be related with scarce literature and less investigation oriented to clinical populations in this range.

Regarding N1 amplitude, results are inconsistent among authors, possibly due to short age range comparison. Our results reveal that $\mathrm{N} 1$ amplitude at $\mathrm{Cz}$ remains stable after adolescence as seen in Fig. 3 (Tsai et al. 2012), but at Fz there is a linear decrease from birth to 60 years old and then an exponential increment (Fig. 4). This result is consistent with the common finding in the literature, that young, adult and elderly differ in their ability to inhibit the processing of task irrelevant information resulting in a higher level of general attention during oddball task (Ford and Pfefferbaum 1991; Friedman et al. 1993; Anderer et al. 1998a; Ford et al. 2001). Furthermore, if we analyze the 

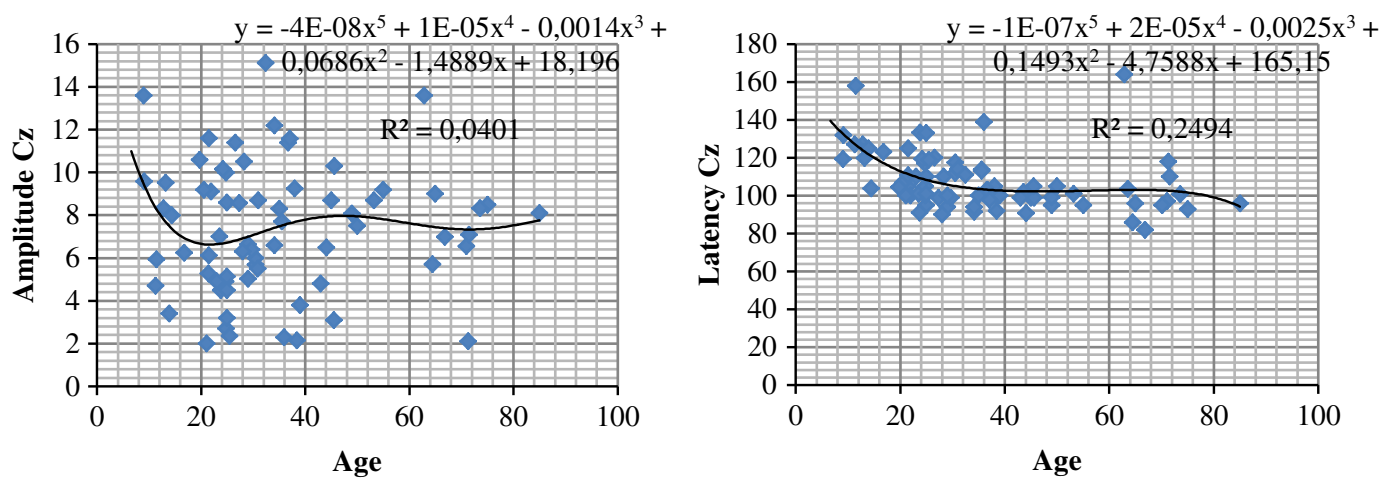

Fig. 3 Scatter plots of the relationship between $\mathrm{N} 1$ amplitude $(\mu \mathrm{V}$; left), latency (ms; right) and age (years) at $\mathrm{Cz}$ (curved lines are the fifth degree polynomial regression over the age range $0-100$ years)
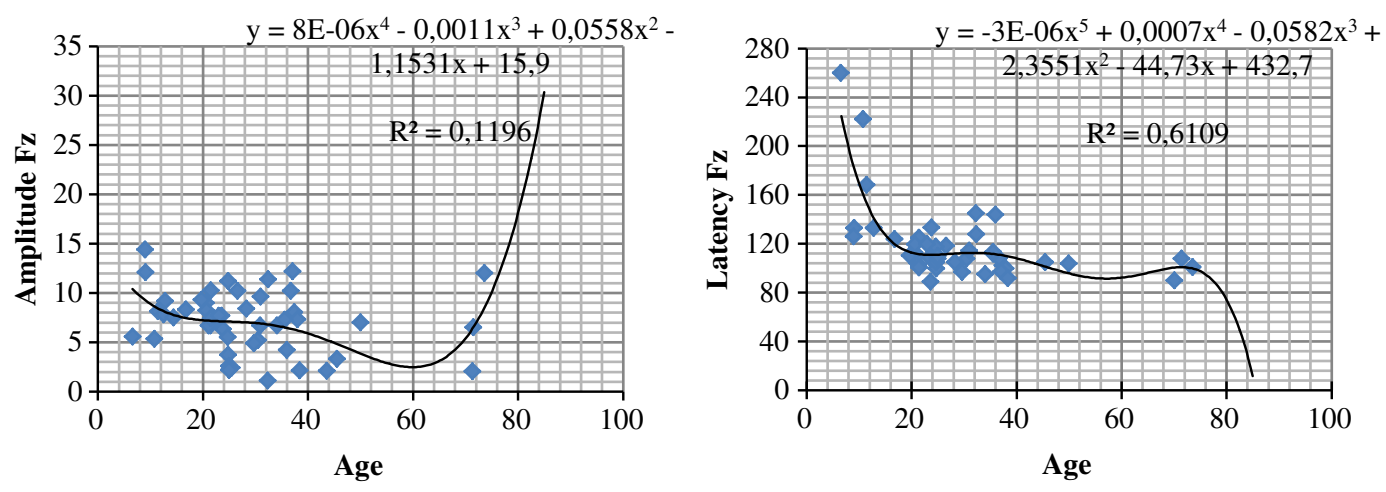

Fig. 4 Scatter plots of the relationship between N1 amplitude ( $\mu \mathrm{V}$; left), latency (ms; right) and age (years) at Fz (curved lines are the fourth and fifth degree, for amplitude and latency, respectively, polynomial regression over the age range $0-100$ years)
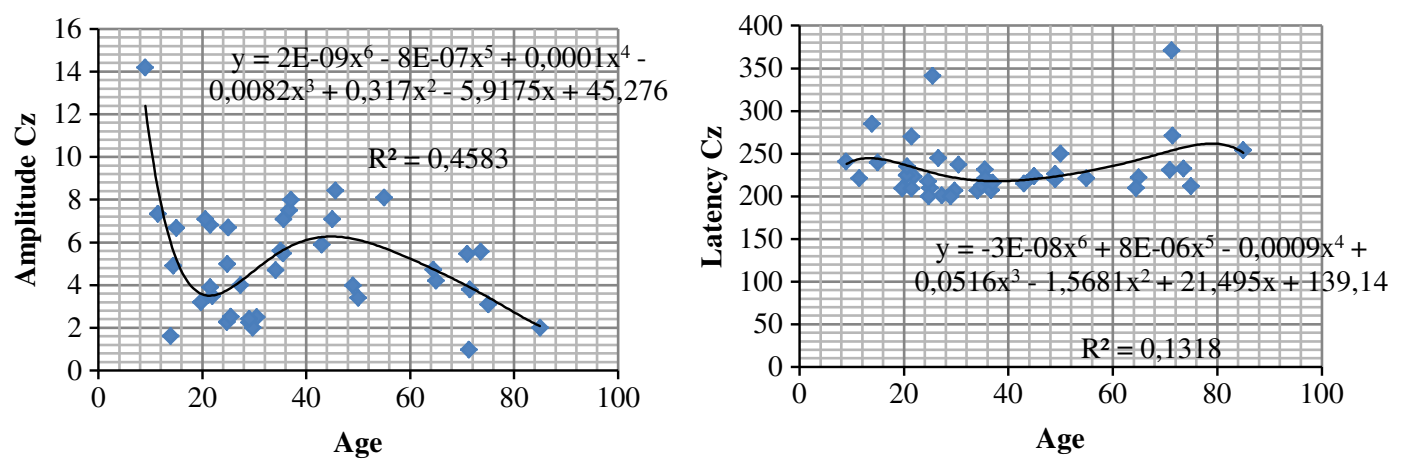

Fig. 5 Scatter plots of the relationship between $\mathrm{N} 2$ amplitude ( $\mu \mathrm{V}$; left), latency (ms; right) and age (years) at $\mathrm{Cz}$ (curved lines are the sixth degree polynomial regression over the age range $0-100$ years)

differences between deviant and standard N1 sources of low resolution electromagnetic tomography (LORETA) studies, younger subjects show a pronounced difference, while it is hard to distinguish deviant N1 from standard N1 source in elder subjects (Pascual-Marqui et al. 1994; Anderer et al. 1998a, b).

This is called the reduction or decline of frontal inhibitory control, due to an ineffective top-down modulation of primary auditory responses by prefrontal cortex (Hasher and Zacks 1988; Čeponienè et al. 2008; for neuro-anatomical evidence see Raz et al. 1997; Chao and Knight, 1997b; Dustman et al. 1996; Kok 1999). In addition, it has been recently found that aging is related with a decrease in event-related spectral power activity, a low phase locking in N1 theta $(4-7 \mathrm{~Hz})$ band over the parietal/frontal regions and with a decrease of functional connections in the alpha 

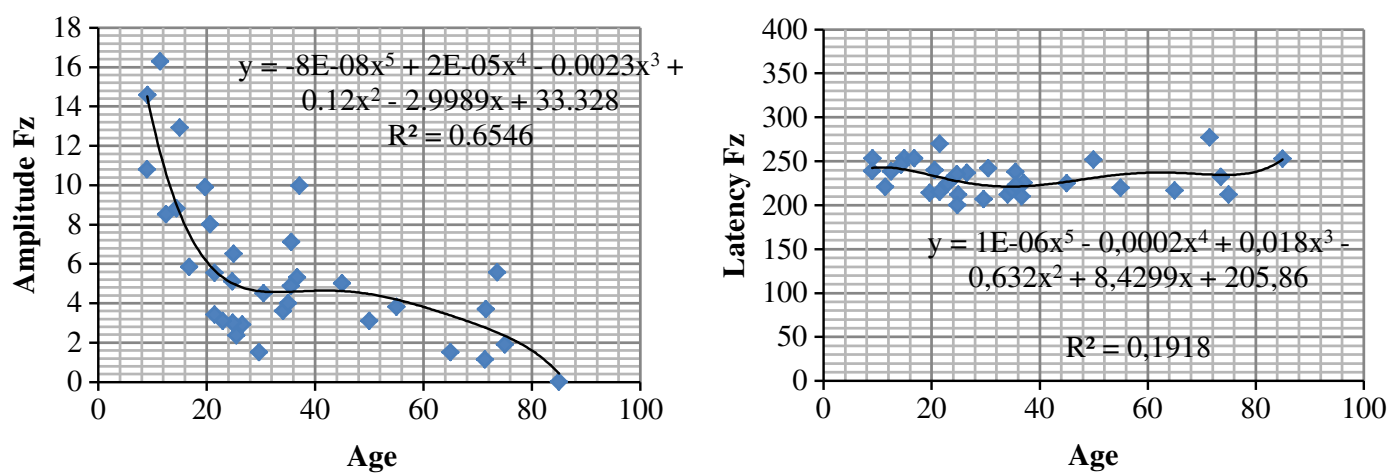

Fig. 6 Scatter plots of the relationship between $\mathrm{N} 2$ amplitude ( $\mu \mathrm{V}$; left), latency (ms; right) and age (years) at Fz (curved lines are the fifth degree polynomial regression over the age range $0-100$ years)

(7-13 Hz) and beta (13-30 Hz) bands (Ho et al. 2012); this might indicate a higher neuroplasticity of younger brains and easy engagement of attentional resources.

Lastly, if we consider and combine results on P3 amplitude decrement and topography change to two distinct foci of activity over age and neuropsychological deficits pointed by several studies, it may be concluded that aging is associated with a general difficulty to distinguish relevant stimulation from irrelevant stimuli, in other words difficulties in selection, categorization and storing in working memory (Picton et al. 1984; Iragui et al. 1993; Lembreghts et al. 1995; Friedman et al. 1997; Amenedo and Díaz 1998; Polich 2007; Martin et al. 2008).

These findings are consistent with $\mathrm{N} 2$ common results. The N2 component is responsible for the classification or categorization of deviant stimuli (Mueller et al. 2008), a previous and required process before storing in working memory. Most studies showed a decrease in the $\mathrm{N} 2$ amplitude and latency from childhood to adulthood (Ladish and Polich 1989; Iragui et al. 1993; Lembreghts et al. 1995; Johnstone et al. 1996; Bertoli and Probst 2005; Mueller et al. 2008; Čeponienè et al. 2008; Tsai et al. 2012), our results are consistent with literature at $\mathrm{Fz}$ and $\mathrm{Cz}$ but only a slight decrement in latency until 40 years old (Figs. 5, 6). Similarly, Goodin et al. (1978) have already found a decrease in N2 latency with age in children of 6-15 years, but an increase in this latency activated or passed as early as during the N1 and P2 components. Regarding adulthood, Picton et al. (1984) reported a significant increase in N2 peak latency with age at a rate of $0.65 \mathrm{~ms}$ per year in adults of 20-79 years, for similar results have been achieved by several authors (Anderer et al. 1996; Mueller et al. 2008). Our results also revealed a slight increment in latency after 40 years old for both $\mathrm{Fz}$ and $\mathrm{Cz}$ (Figs. 5, 6). The increase in N2 latency seems to be due to linear increases in one of its components' latency (N2b) at Fz, $\mathrm{Cz}$ and $\mathrm{Pz}$, indicating that the aging-related slowing begins at controlled memory comparison between non-target and target stimuli (Amenedo and Díaz 1998).
Keeping the above in mind, N2 amplitude decrement over age and latency increment after adulthood are the only auditory ERP findings that are in agreement with agerelated decrease in auditory acuity (Baltes and Lindenberger 1997; Chao and Knight 1997a, b; Tremblay et al. 2003; Čeponienè et al. 2008). However, other studies have found no changes in N2 amplitude with advancing age (Brown et al. 1983; Picton et al. 1984; Barrett et al. 1987) and even increases at central and parietal scalp sites (Iragui et al. 1993; Enoki et al. 1993) which might explain N1 and $\mathrm{N} 2$ amplitude increment at $\mathrm{Cz}$ from 20 to 45 years in our scatter-plot (Figs. 3, 5). N1 and N2 amplitude increase in this range (20-45 years) might correlate with growth of synaptic density, paralleled by improving synaptic efficiency and spatiotemporal synchronization.

Further, Čeponiene et al. (2008) consider that N2 diminution with age could represent cyto-architectonic derangement in sensory regions, including diminished synaptic synchronization causing decreased processing speed (Peters 2002).

Finally, to consider using N1 and N2 components in clinical practice, it must be kept in mind that ERPs change topography over age, due to senescent-related changes and individual profound cortical reorganization. Regarding N1 and N2, a change from frontal to central-parietal topography peak with aging is the most common finding (Anderer et al. 1996, 1998a; Amenedo and Díaz 1998; Mueller et al. 2008) because frontal areas have also been reported to be more affected by aging than others (Goodin et al. 1978; West 1996; Amenedo and Díaz 1998; Mueller et al. 2008). Anderer et al. (1996) reported that N1 latency increases with advancing age were dependent on electrode position, as they only increased at posterior scalp sites. However, gender is a variable that should be taken notice of. Some authors have reported differences across electrode location and amplitude (Gölgeli et al. 1999). Very few studies reported control groups by gender precluding us to include it in our statistical analyses. In addition, a 
comprehensive review regarding possible effects of IQ and years of education is of particular importance, in a forthcoming article. Also, despite means and standard deviations for $\mathrm{N} 1$ and $\mathrm{N} 2$ in Table 3, it is still a challenge to compare results of a single participant, and does not exempt of establishing normative values in the local laboratory, department or service.

It is important to conduct systematic reviews and normative values regarding $\mathrm{P} 1$ and $\mathrm{P} 2$ components and $\mathrm{P} 1-$ N1-P2 complex (Zheng et al. 2011) also with auditory oddball paradigms and considering putative gender differences. The objective measurement of central auditory processing and integrity of final auditory pathway should not depend on just one component but on the presence of several ERPs (complex), reflecting progressive central sound processing and representation (McGee et al. 1997; Näätänen et al. 2007; Nikjeh et al. 2009) and also preservation of neuronal synchrony encoding temporal information (Rance et al. 2002; Tomé et al. 2012).

\section{Summary and conclusion}

The clinical use of $\mathrm{N} 1$ and $\mathrm{N} 2$ remains minimal because recording requires special equipment and knowledge to process and extract ERPs. But also because normative data are lacking and responses vary widely between subjects.

Results from this systematic review suggest that $\mathrm{Fz}$ and $\mathrm{Cz}$ are the electrode locations to clinically consider the normative values presented in results section. However, to accept N1 and N2 components as brain markers of preattentive and early cognitive function, it should be regarded parallel changes in brain development and components topography over age, mainly in childhood, adolescence and after 60 years. But certainly, using auditory oddball paradigms in an attended or unattended condition, N1 and N2 components seem a promising clinical indexing measure in the assessment of central auditory processing among several clinical conditions and populations.

Conflict of interest The authors have declared that there are no conflicts of interest in relation to the subject of this study. The contribution of Kamila Nowak was supported by NCBR grant No. INNOTECH-K1/IN1/30/159041/NCBR/12

\section{References}

Akaho R (1996) The effects of antiepileptic drugs on cognition in normal volunteers. Psychiatry Clin Neurosci 50:60-61

Altieri N (2013) Neural dynamics of audiovisual integration for speech and non-speech stimuli: a psychophysical study. Open Neurosci J 7:5-18

Amenedo E, Díaz F (1998) Aging-related changes in processing of non-target and target stimuli during an auditory oddball task. Biol Psychol 48:235-267
Anderer P, Semlitsch HV, Saletu B (1996) Multichannel auditory event-related brain potentials: effects of normal aging on the scalp distribution of N1, P2, N2 and P300 latencies and amplitudes. Electroencephalogr clin Neurophysiol 99:458-472

Anderer P, Pascual-Marqui RD, Semlitsch HV, Saletu B (1998a) Differential effects of normal aging on sources of standard N1, target N1 and target P300 auditory event-related brain potentials revealed by low resolution electromagnetic tomography (LORETA). Electroencephalogr clin Neurophysiol 108:160-174

Anderer P, Pascual-Marqui RD, Semlitsch HV, Saletu B (1998b) Electrical sources of $\mathrm{P} 300$ event-related brain potentials revealed by low resolution electromagnetic tomography. 1. Effect of normal aging. Neuropsychobiology 37:20-27

Baltes PB, Lindenberger U (1997) Emergence of a powerful connection between sensory and cognitive functions across the adult life span: a new window to the study of cognitive aging? Psychol Aging 12(1):12-21

Barrett G, Neshige R, Shibasaki H (1987) Human auditory and somatosensory event-related potentials: effects of response condition and age. Electroencephalogr Clin Neurophysiol 66:409-417

Barry RJ, Kirkaikul S, Hodder D (2000) EEG alpha activity and the ERP to target stimuli in an auditory oddball paradigm. Int $\mathbf{J}$ Psychophysiol 39:39-50

Barry RJ, de Pascalis V, Hodder D, Clarke AR, Johnstone SJ (2003) Preferred EEG brain states at stimulus onset in a fixed interstimulus interval auditory oddball task, and their effects on ERP components. Int J Psychophysiol 47:187-198

Barry RJ, Rushby JA, Johnstone SJ, Clarke AR, Croft RJ, Lawrence CA (2004) Preferred EEG brain states at stimulus onset in a fixed interstimulus interval auditory oddball task, and their effects on ERP components. Clin Neurophysiol 115:2593-2601

Barry RJ, Rushby JA, Smith JL, Clarke AR, Croft RJ (2006) Dynamics of narrow-band EEG phase effects in the passive auditory oddball task. Eur J Neurosci 24:291-304

Bennett M, Hacker P (2007) Philosophical foundations of neuroscience. In: Bennett M, Dennett D, Hacker P, Searle J (eds) Neuroscience and philosophy: brain, mind and language, 1st edn. Columbia University Press, New York, pp 3-49

Bentin S, Carmon A (1984) Verbal information processing and hemispheric AEP asymmetry. Ann N Y Acad Sci 425:166-170

Berger H (1929) Uber das Elektrenkephalogramm das menchen. Archiv fur Psychiatrie 87:527-570

Bertoli S, Probst R (2005) Lack of standard N2 in elderly participants indicates inhibitory processing deficit. NeuroReport 16(17):1933-1937

Bortoletto M, Tona G, Scozzari S, Sarasso S, Stegagno L (2011) Effects of sleep deprivation on auditory change detection: a N1Mismatch Negativity study. Int J Psychophysiol 81:312-316

Boucher O, Bastien C, Saint-Amour D, Dewailly E, Ayotte P, Jacobson J et al (2010) Prenatal exposure to methylmercury and PCBs affects distinct stages of information processing: an eventrelated potential study with Inuit children. Neurotoxicology 31:373-384

Brigham J, Moss HB, Murrelle EL, Kirisci L, Spinelli JS (1997) Event-related potential negative shift in sons of polysubstanceand alcohol-use disorder fathers. Psych Res 73:133-146

Brown WS, Marsh JT, La Rue A (1983) Exponential electrophysiological aging: P300 latency. Electroencephalogr Clin Neurophysiol 55:277-285

Brown KJ, Gonsalvez CJ, Harris AWF, Williams LM, Gordon E (2002) Target and non-target ERP disturbances in first episode vs. chronic schizophrenia. Clin Neurophysiol 113:1754-1763

Brown CR, Clarke AR, Barry RJ (2006) Inter-modal attention: ERPs to auditory targets in an inter-modal oddball task. Int $\mathrm{J}$ Psychophysiol 62:77-86 
Bruneau N, Roux S, Guérin P, Barthélémy C, Lelord G (1997) Temporal prominence of auditory evoked potentials (N1 wave) in 4-8 year-old children. Psychophysiology 34:32-38

Cahn B, Polich J (2009) Meditation (Vipassana) and the P3a eventrelated brain potential. Int J Psychophysiol 72:51-60

Cansino S, Williamson SJ, Karron D (1994) Tonotopic organization of human auditory association cortex. Brain Res 663:38-50

Čeponienė R, Rinne T, Näätänen R (2002) Maturation of cortical sound processing as indexed by event-related potentials. Clin Neurophysiol 108:870-882

Čeponienè R, Lepistö T, Alku P, Aro H, Näätänen R (2003) Eventrelated potential indices of auditory vowel processing in 3-yearold children. Clin Neurophysiol 114:652-661

Čeponiene R, Westerfield M, Torki M, Townsend J (2008) Modalityspecificity of sensory aging in vision and audition: evidence from event-related potentials. Brain Res 1215:53-68

Chao LL, Knight RT (1997a) Age-related prefrontal alterations during auditory memory. Neurobiol Aging 18(1):87-95

Chao LL, Knight RT (1997b) Prefrontal deficits in attention and inhibitory control with aging. Cereb Cortex 7(1):63-69

Chao LL, Lindgren JA, Flenniken DL, Weiner WW (2004) ERP evidence of impaired central nervous system function in virally suppressed HIV patients on antiretroviral therapy. Clin Neurophysiol 115:1583-1591

Chunhau P, Shimono M, Iwanaga M, Hasegawa R, Honda Y, Shirahata A et al (2005) Analysis of P3 in a continuous 40-min auditory oddball task. Int Cong Series 1278:105-108

Cohen HL, Ji J, Chorlian DB, Begleiter H, Porjesz B (2002) Alcoholrelated ERP changes recorded from different modalities: a topographic analysis. Alcohol Clin Exp Res 26(3):303-317

Courchesne E (1990) Chronology of postnatal human brain development: event-related potential, positron emission tomography, myelinogenesis, and synaptogenesis studies. In: Rohrbaugh JW, Parasuraman R, Johnson R (eds) Event-related brain potentials: basic issues and applications. Oxford University Press, New York, pp 210-241

Crowley KE, Colrain IM (2004) A review of the evidence for P2 being an independent component process: age, sleep and modality. Clin Neurophysiol 115:732-744

Dassanayake T, Gawarammana IB, Weerasinghe V, Dissanayake PS, Pragaash S, Dawson A, Senanayake N (2009) Auditory eventrelated potential changes in chronic occupational exposure to organophosphate pesticides. Clin Neurophysiol 120:1693-1698

Davis PA (1939) Effects of acoustic stimuli on the waking human brain. J Neurophysiology 2:494-499

Davis H, Zerlin S (1966) Acoustic relations of the human vertex potential. J Acoust Soc Am 39:109-116

Demiralp T, Yordanova J, Kolev V, Ademoglu A, Devrim M, Samar VJ (1999) Time-frequency analysis of single-sweep eventrelated potentials by means of fast wavelet transform. Brain Lang 66:129-145

Dixit A, Vaney N, Tandon OP (2006) Evaluation of cognitive brain functions in caffeine users: a P3 evoked potential study. Indian J Physiol Pharmacol 50(2):175-180

Donchin E (1981) Presidential address, 1980. Surprise!. Surprise? Psychophysiology 18:493-513

Duncan C, Barry R, Connolly J, Fischer C, Michie P, Näätänen R et al (2009) Event-related potentials in clinical research: guidelines for eliciting, recording, and quantifying mismatch negativity, P300, and N400. Clin Neurophysiol 120:1883-1908

Dustman RE, Emmerson RY, Shearer DE (1996) Life span changes in electrophysiological measures of inhibition. Brain Cogn 30(1):109-126

Eggermont JJ (1988) On the rate of maturation of sensory evoked potentials. Electroencephalogr Clin Neurophysiol 70(4):293-305
Elberling C, Bak C, Kofoed B, Lebech J, Saermark K (1980) Magnetic auditory responses from the human brain. A preliminary report. Scand Audiol 9:185-190

Enoki H, Sanada S, Yoshinaga H, Oka E, Ohtahara S (1993) The effects of age on the N200 component of the auditory eventrelated potentials. Cogn Brain Res 1:161-167

Eysenck MW, Keane MT (2003) (eds) Cognitive psychology: a student's handbook, 4th edn. Psychology Press, East Sussex, pp 1-27

Fabiani M, Gratton G, Federmeier (2007) Event-related brain potentials: methods, theory, and applications. In: Cacioppo J, Tassinary LG, Berntson GG (eds) The Handbook of Psychophysiology, 3rd edn. Cambridge University Press, New York, pp 94-119

Ford JM, Pfefferbaum A (1991) Event-related potentials and eyeblink responses in automatic and controlled processing: effects of age. Electroencephalogr Clin Neurophysiol 78:361-377

Ford JM, Mathalon DH, Kalba S, Marsh L, Pfefferbaum A (2001) N1 and P300 abnormalities in patients with schizophrenia, epilepsy, and epilepsy with schizophrenia like features. Biol Psychiatry 49:848-860

Friedman D, Simpson GV, Hamberger M (1993) Age-related changes in scalp topography to novel and target stimuli. Psychophysiology 30:383-396

Friedman D, Kazmerski V, Fabiani M (1997) An overview of agerelated changes in the scalp distribution of P3b. Electroencephalogr Clin Neurophysiol 104:498-513

Gandelman-Marton R, Theitler J, Klein C, Rabey JM (2010) The effects of immediate and short-term retest on the latencies and amplitudes of the auditory event-related potentials in healthy adults. J Neurosci Methods 186:77-80

Gilley PM, Sharma A, Dorman M, Martin K (2005) Developmental changes in refractoriness of the cortical auditory evoked potential. Clin Neurophysiol 116:646-657

Gilmore CS, Clementz BA, Buckley PF (2005) Stimulus sequence affects schizophrenia-normal differences in event processing during an auditory oddball task. Cogn Brain Res 24:215-227

Gilmore CS, Clementz BA, Berg P (2009) Hemispheric differences in auditory oddball responses during monaural versus binaural stimulation. Int J Psychophysiol 73:326-333

Gölgeli A, Süer C, Özesmi Ç, Dolu N, Aşcioğ lu M, Şahin Ö (1999) The effect of sex differences in young adults on event-related potentials. Int J Neurosci 99:69-77

Gonsalvez CJ, Gordon E, Grayson S, Barry RJ, Lazzaro I, Bahramali $\mathrm{H}$ (1999) Is the target-to-target interval a critical determinant of P3 amplitude? Psychophysiology 36:643-654

Goodin DS, Squires KC, Henderson BH, Starr A (1978) Age-related variations in evoked potentials to auditory stimuli in normal human subjects. Electroencephalogr Clin Neurophysiol 44:447-458

Guney F, Genc B, Kutlu R, Ilhan B (2009) Auditory P300 eventrelated potential in tobacco smokers. J Clin Neurosci 16:1311-1315

Haig AR, Rennie C, Gordon E (1997) The use of Gaussian component modelling to elucidate average ERP component overlap in schizophrenia. J Psychophysiol 11:173-187

Hamm JP, Ethridge LE, Shapiro JR, Pearlson GD, Tamminga CA, Sweeney JA, Keshavan MS, Thaker GK, Clementz BA (2013) Family history of psychosis moderates early auditory cortical response abnormalities in non-psychotic bipolar disorder. Bipolar Disord 15:774-786

Hari R, Aittoniemi K, Järvinen M-L, Katila T, Varpula T (1980) Auditory evoked transient and sustained magnetic fields of the human brain. Localization of neural generators. Exp Brain Res 40:237-240 
Hasher L, Zacks RT (1988) Working memory, comprehension, and aging: a review and new view. In: Bower GH (ed) The Psychology of Learning and Motivation, 22. Academic Press, San Diego, pp 193-225

Hegerl U, Gaebel W, Gutzman H, Ulrich G (1988) Auditory evoked potentials as possible predictors of outcome in schizophrenic outpatients. Int J Psychophysiol 6:207-214

Henkin Y, Kishon-Rabin L, Gadoth N, Pratt H (2002) Auditory eventrelated potentials during phonetic and semantic processing in children. Audiol Neurootol 7:228-239

Hillyard SA, Kutas M (1983) Electrophysiology of cognitive processing. Ann Rev Psychol 34:33-61

Hillyard SA, Hink RF, Schwent VL, Picton TW (1973) Electrical signs of selective attention in the human brain. Science 182:177-180

Hirata K, Katayama S, Yamazaki K, Fujikane M, Katayama K (1996) Electric field distribution of event-related potentials in stroke patients. Brain Topogr 8(3):279-284

Ho M-C, Chou C-Y, Huang C-F, Lin Y-T, Shih C-S, Han S-Y, Shen M-H et al (2012) Age-related changes of task-specific brain activity in normal aging. Neurosci Lett 507:78-83

Huttenlocher PR (1979) Synaptic density in human frontal cortexdevelopmental changes and effects of aging. Brain Res 163(2):195-205

Inui K, Okamoto H, Miki K, Gunji A, Kakigi R (2006) Serial and parallel processing in the human auditory cortex: a magnetoencephalographic study. Cereb Cortex 16:18-30

Iragui VJ, Kutas M, Mitchiner MR, Hillyard SA (1993) Effects of aging on event-related brain potentials and reaction times in an auditory oddball task. Psychophysiology 30:10-22

Jääskeläinen IP (2012) Introduction to cognitive neuroscience. Ventus Publishing ApS, Denmark, pp 92-112. http://pt.scribd.com/doc/ 102335497/Introduction-to-Cognitive-Neuroscience

Jääskeläinen IP, Ahveninen J, Bonmassar G, Dale AM, Ilmoniemi RJ, Levänen $S$ et al (2004) Human posterior auditory cortex gates novel sounds to consciousness. Proc Nat Acad Sci (USA) 101:6809-6814

Johnstone SJ, Barry RJ, Anderson JW, Coyle SF (1996) Age-related changes in child and adolescent event-related potential component morphology, amplitude and latency to standard and target stimuli in an auditory oddball task. Int J Psychophysiol 24:223-238

Johnstone SJ, Barry RJ, Anderson JW (2001) Topographic distribution and developmental timecourse of auditory event-related potentials in two subtypes of attention-deficit hyperactivity disorder. Int J Psychophysiol 42:73-94

Kazis A, Kimiskidis V, Georgiadis G, Kapinas K (1996) Cognitive event-related potentials and magnetic resonance imaging in myotonic dystrophy. Neurophysiol Clin 26:75-84

Kok A (1999) Varieties of inhibition: manifestations in cognition, event-related potentials and aging. Acta Psychol (Amst) 101(2-3):129-158

Kreukels BPC, Hamburger HL, de Ruiter MB, van Dam FS, Ridderinkhof KR, Boogerd W, Schagen SB (2008) ERP amplitude and latency in breast cancer survivors treated with adjuvant chemotherapy. Clin Neurophysiol 119:533-541

Ladish C, Polich J (1989) P300 and probability in children. J Exp Child Psychol 48:212-223

Lembreghts M, Crasson M, EL Ahmadi A, Timsit-Berthier M (1995) Éude de la variabilité interindividuelle des potentiels évoqués auditifs exogènes et endogènes en condition d'attention volontaire. Neurophysiol Clin 25:203-223

Liasis A, Bamiou DE, Boyd S, Towell A (2006) Evidence for a neurophysiologic auditory deficit in children with benign epilepsy with centro-temporal spikes. J Neural Transm 113:939-949
Lucchesi L, Pompéia S, Manzano G, Kohn A, Galduroz J, Bueno O, Tufik T (2003) Flunitrazepam-induced changes in neurophysiological, behavioural, and subjective measures used to assess sedation. Prog Neuro-Psychopharmacol Biol Psychiatry 27:525-533

Martin BA, Tremblay KL, Korczak P (2008) Speech evoked potentials: from the laboratory to the clinic. Ear Hear 29:285-313

May P, Tiitinen H (2010) Mismatch negativity (MMN), the devianceelicited auditory deflection, explained. Psychophysiology 47:66-122

McGee T, Kraus N, Nicol T (1997) Is it really a mismatch negativity? An assessment of methods for determining response validity in individual subjects. Electroencephalogr Clin Neurophysiol 104:359-368

Moher D, Liberati A, Tetzlaff J, Altman DG (2009) Preferred reporting items for systematic reviews and meta-analyses: the PRISMA statement. Ann Intern Med 151(4):264-269

Mueller V, Brehmer Y, von Oertzen T, Li S-C, Lindenberger U (2008) Electrophysiological correlates of selective attention: a lifespan comparison. BMC Neurosci 9:18

Mulert C, Jäger L, Schmitt R, Bussfeld P, Pogarell O, Möller H-J et al (2004) Integration of fMRI and simultaneous EEG: towards a comprehensive understanding of localization and time-course of brain activity in target detection. Neuroimage 22:83-94

Murray MM, Brunet D, Michel CM (2008) Topographic ERP analyses: a step-by-step tutorial review. Brain Top 20(4):249-264

Näätänen R (1975) Selective attention and evoked potentials in humans-a critical review. Biol Psychol 2:237-307

Näätänen R (1982) Processing negativity: an evoked-potential reflection of selective attention. Psychol Bull 92:605-640

Näätänen R, Picton T (1987) The N1 wave of the human electric and magnetic response to sound: a review and an analysis of the component structure. Psychophysiology 24:375-425

Näätänen R, Gaillard AWK, Mäntysalo S (1978) Early selective attention effect on evoked potential reinterpreted. Acta Psychol (Amst) 42:313-329

Näätänen R, Paavilainen P, Rinne T, Alho K (2007) The mismatch negativity (MMN) in basic research of central auditory processing: a review. Clin Neurophysiol 118:2544-2590

Neuhaus AH, Popescu FC, Rentzsch J, Gallinat J (2013) Critical evaluation of auditory event-related potential deficits in schizophrenia: evidence from large-scale single-subject pattern classification. Schizophr Bull doi:10.1093/schbul/sbt151

Nikjeh DA, Lister JJ, Frisch SA (2009) Pre-attentive cortical-evoked responses to pure tones, harmonic tones, and speech: influence of music training. Ear Hear 30:43-46

Novak GP, Ritter W, Vaughan HG Jr, Wiznitzer ML (1990) Differentiation of negative event-related potentials in an auditory discrimination task. Electroencephalogr Clin Neurophysiol $75: 255-275$

Novak GP, Ritter W, Vaughan HG Jr (1992) Mismatch detection and the latency of temporal judgements. Psychophysiology 29:399-411

Ogawa T, Tanaka H, Hirata K (2009) Cognitive deficits in amyotrophic lateral sclerosis evaluated by event-related potentials. Clin Neurophysiol 120:659-664

Pantev C, Bertrand O, Eulitz C, Verkindt C, Hampson S, Schuierer G et al (1995) Specific tonotopic organizations of different areas of the human auditory cortex revealed by simultaneous magnetic and electric recordings. Electroencephalogr clin Neurophysiol 94:26-40

Papanicolaou AC, Baumann S, Rogers RL, Saydjari C, Amparo EG, Eisenberg HM (1990) Localization of auditory response sources 
using magnetoencephalography and magnetic resonance imaging. Arch Neurol 47:33-37

Pascual-Marqui RD, Michel CM, Lehmann D (1994) Low resolution electromagnetic tomography: a new method for localizing electrical activity in the brain. Int J Psychophysiol 18:49-65

Peters A (2002) Structural changes in the normally aging cerebral cortex of primates. Prog Brain Res 136:455-465

Picton TW, Stuss DT, Champagne SC, Nelson RF (1984) The effects of age on human event-related potentials. Psychophysiology $21: 312-325$

Polich J (2007) Updating P300: an integrative theory of P3a and P3b. Clin Neurophysiol 118:2128-2148

Ponton CW, Moore JK, Eggermont JJ (1999) Prolonged deafness limits auditory system developmental plasticity: evidence from evoked potentials study in children with cochlear implants. Scand Audiol Suppl 51:13-22

Ponton CW, Eggermont JJ, Kwong B, Don M (2000) Maturation of human central auditory system activity: evidence from multichannel evoked potentials. Clin Neurophysiol 111:220-236

Potts G, Hirayasu Y, O’Donnell B, Shenton M, McCarley R (1998a) High-density recording and topographic analysis of the auditory oddball event-related potential in patients with schizophrenia. Biol Psychiatry 44:982-989

Potts G, Dien J, Hartry-Speiser A, McDougal L, Tucker D (1998b) Dense sensor array topography of the event-related potential to task-relevant auditory stimuli. Electroencephalogr clin Neurophysiol 106:444-456

Rance G, Cone-Wesson B, Wunderlich J, Dowell R (2002) Speech perception and cortical event related potentials in children with auditory neuropathy. Ear Hear 23:239-253

Raz N, Gunning FM, Head D, Dupuis JH, McQuain J, Briggs SD et al (1997) Selective aging of the human cerebral cortex observed in vivo: differential vulnerability of the prefrontal gray matter. Cereb Cortex 7(3):268-282

Reinvang I, Nordby H, Nielsen CS (2000) Information processing deficits in head injury assessed with ERPs reflecting early and late processing stages. Neuropsychologia 38:995-1005

Ritter W, Vaughan HG (1969) Averaged evoked responses in vigilance and discrimination: a reassessment. Science 164:326-328

Ritter W, Simson R, Vaughan H, Friedman D (1979) A brain event related to the making of a sensory discrimination. Science 203:1358-1361

Rosburg T, Boutros NN, Ford JM (2008) Reduced auditory evoked potential component N100 in schizophrenia-a critical review. Psychiatry Res 161:259-274

Sakamoto K, Nakata H, Kakigi R (2009) The effect of mastication on human cognitive processing: a study using event-related potentials. Clin Neurophysiol 120:41-50

Sams M, Paavilainen P, Alho K, Näätänen R (1985) Auditory frequency discrimination and event-related potentials. Electroencephalogr Clin Neurophysiol 62:437-448

Schneider A, Leigh MJ, Adams P, Nanakul R, Chechi T, Olichney J, Hagerman R, Hessl D (2013) Electrocortical changes associated with minocycline treatment in fragile $\mathrm{X}$ syndrome. J Psychopharmacol 27(10):956-963

Segalowitz S, Bernstein D, Lawson S (2001) P300 event-related potential decrements in well-functioning university students with mild head injury. Brain Cogn 45:342-356

Sharma A, Kraus N, McGee TJ, Nicol TG (1997) Developmental changes in $\mathrm{P} 1$ and $\mathrm{N} 1$ central auditory responses elicited by consonant-vowel syllables. Electroencephalogr clin Neurophysiol 104:540-545

Shelley A-M, Silipo G, Javitt D (1999) Diminished responsiveness of ERPs in schizophrenic subjects to changes in auditory stimulation parameters: implications for theories of cortical dysfunction. Schizophr Res 37:65-79

Siedenberg R, Goodin DS, Aminoff MJ, Rowley HA, Roberts TPL (1996) Comparison of late components in simultaneously recorded event-related electrical potentials and event-related magnetic fields. Electroencephalogr clin Neurophysiol 99:191-197

Squires NK, Squires KC, Hillyard SA (1975) Two varieties of longlatency positive waves evoked by unpredictable auditory stimuli in man. Electroencephalogr clin Neurophysiol 38(4):387-401

Sumi N, Nan'no H, Fujimoto O, Ohta Y, Takeda M (2000) Interpeak latency of auditory event-related potentials (P300) in senile depression and dementia of the Alzheimer type. Psychiatry Clin Neurosci 54(6):679-684

Tanaka H, Arai M, Harada M, Hozumi A, Hirata K (2012) Cognition and event-related potentials in adult-onset non-demented myotonic dystrophy type 1. Clin Neurophysiol 123:261-269

Tarter R, Blackson T, Brigham J, Moss H, Caprara G (1995) The association between childhood irritability and liability to substance use in early adolescence: a 2-year follow-up study of boys at risk for substance abuse. Drug Alcohol Depend 39:253-261

Tiitinen H, May P, Reinikainen K, Näätänen R (1994) Attentive novelty detection in humans is governed by pre-attentive sensory memory. Nature 372:90-92

Tomé D, Marques-Teixeira J, Barbosa F (2012) Temporal lobe epilepsy in childhood-a study model of auditory processing. J Neurol Neurophysiol 3(2). doi:10.4172/2155-9562.1000123

Toscano J, McMurray B, Dennhardt J, Luck S (2010) Continuous perception and graded categorization: electrophysiological evidence for a linear relationship between the acoustic signal and perceptual encoding of speech. Psychological Sci 21(10):1532-1540

Tremblay KL, Piskosz M, Souza P (2003) Effects of age and agerelated hearing loss on the neural representation of speech cues. Clin Neurophysiol 114(7):1332-1343

Tsai M-L, Hung K-L, Tung W, Chiang T-R (2012) Age-changed normative auditory event-related potential value in children in Taiwan. J Form Med Assoc 111:245-252

Ullsperger P, Freude G, Erdmann U (2001) Auditory probe sensitivity to mental workload changes-an event-related potential study. Int J Psychophysiol 40:201-209

Valkonen-Korhonen M, Purhonena M, Tarkka I, Sipilä P, Partanen J, Karhu J et al (2003) Altered auditory processing in acutely psychotic never-medicated first-episode patients. Cogn Brain Res 17:747-758

van Dinteren R, Arns M, Jongsma MLA, Kessels RPC (2014) P300 development across the lifespan: a systematic review and metaanalysis. PLoS One 9(2):e87347

van Harten B, Laman DM, van Duijn H, Knol DL, Stam CJ, Scheltens P, Weinstein HC (2006) The auditory oddball paradigm in patients with vascular cognitive impairment: a prolonged latency of the N2 complex. Dement Geriatr Cogn Disord 21:322-327

Verma N, Nichols C, Greiffenstein M, Singh R, Hurst-Gordon D (1989) Waves earlier than P3 are more informative in putative subcortical dementias: a study with mapping and neuropsychological techniques. Brain Topogr 1(3):183-191

Wang W, Wang Y-H (2001) Sensation seeking correlates of passive auditory P3 to a single stimulus. Neuropsychologia 39:1188-1193

Wang W, Datta H, Sussman E (2005) The development of the length of the temporal window of integration for rapidly presented auditory information as indexed by MMN. Clin Neurophysiol 116:1695-1706

West RL (1996) An application of prefrontal cortex function theory to cognitive aging. Psychol Bull 120:272-292 
Wetzel N, Widmann A, Schröger E (2011) Processing of novel identifiability and duration in children and adults. Biol Psychol 86:39-49

Whelan R, Lonergan R, Kiiski H, Nolan H, Kinsella K, Bramham J, O'Brien M et al (2010) A high-density ERP study reveals latency, amplitude, and topographical differences in multiple sclerosis patients versus controls. Clin Neurophysiol 121:1420-1426

Winkler I (2007) Interpreting the mismatch negativity. J Psychophysiol 21(3-4):147-163

Winter O, Kok A, Kenemans J, Elton M (1995) Auditory eventrelated potentials to deviant stimuli during drowsiness and stage 2 sleep. Electroencephalogr clin Neurophysiol 96:398-412

Wise V, McFarlane AC, Clark CR, Battersby M (2009) Event-related potential and autonomic signs of maladaptive information processing during an auditory oddball task in panic disorder. Int J Psychophysiol 74:34-44

Wolpaw JR, Penry JK (1975) A temporal component of the auditory evoked response. Electroencephalogr clin Neurophysiol 39(6):609-620

Woods DL, Alho K, Algazi A (1993) Intermodal selective attention: evidence for processing in tonotópica auditory fields. Psychophysiology 30:287-295

Wright MJ, Geffen GM, Geffen LB (1996) ERP measures of stimulus processing during an auditory oddball task in Parkinson's disease: evidence for an early information processing deficit. Parkinsonism Rel Disord 2(1):13-21

Yamamoto T, Williamson SJ, Kaufman L, Nicholson C, Llinás R (1988) Magnetic localization of neuronal activity in the human brain. Proc Nat Acad Sci (USA) 85:8732-8736

Yvert B, Crouzeix A, Bertrand O, Seither-Preisler A, Pantev C (2001) Multiple supratemporal sources of magnetic and electric auditory evoked middle latency components in humans. Cereb Cortex 11:411-423

Yvert B, Fischer C, Bertrand O, Pernier J (2005) Localization of human supratemporal auditory areas from intracerebral auditory evoked potentials using distributed source models. Neuroimage 28:140-153

Zheng Y, Zhao F, Liang M, Bardsley B, Yang H, Zhang Z (2011) Toward an understanding of auditory evoked cortical eventrelated potentials: characteristics and classification. Audiol Med 1-10. doi:10.3109/1651386X.2010.537910

Zhu W, Zhang J, Liu H, Ding X, Ma Y, Zhou C (2008) Differential cognitive responses to guqin music and piano music in Chinese subjects: an event-related potential study. Neurosci Bull 24(1):21-28 